\title{
SPECTRAL GAP LOWER BOUND FOR THE ONE-DIMENSIONAL FRACTIONAL SCHRÖDINGER OPERATOR IN THE INTERVAL
}

\author{
KAMIL KALETA
}

\begin{abstract}
We prove the uniform lower bound for the difference $\lambda_{2}-\lambda_{1}$ between first two eigenvalues of the fractional Schrödinger operator $(-\Delta)^{\alpha / 2}+V$, which is related to the Feynman-Kac semigroup of the symmetric $\alpha$-stable process killed upon leaving open interval $(a, b) \in \mathbf{R}$ with symmetric differentiable single-well potential $V$ in the interval $(a, b), \alpha \in(1,2)$. "Uniform" means that the positive constant appearing in our estimate $\lambda_{2}-\lambda_{1} \geq C_{\alpha}(b-a)^{-\alpha}$ is independent of the potential $V$. In general case of $\alpha \in(0,2)$, we also find uniform lower bound for the difference $\lambda_{*}-\lambda_{1}$, where $\lambda_{*}$ denotes the smallest eigenvalue related to the antisymmetric eigenfunction $\varphi_{*}$. We discuss some properties of the corresponding ground state eigenfunction $\varphi_{1}$. In particular, we show that it is symmetric and unimodal in the interval $(a, b)$. One of our key argument used in proving the spectral gap lower bound is some integral inequality which is known to be a consequence of the Garsia-Rodemich-Rumsey-Lemma.
\end{abstract}

\section{Introduction AND STATEMENT OF RESUlts}

The main purpose of this paper is to prove an uniform lower bound for the spectral gap of the fractional Schrödinger operator with symmetric differentiable single-well potential on a bounded interval of the real line. Such an operator is related to the Feynman-Kac semigroup of the killed symmetric $\alpha$-stable process. To obtain this bound we study some basic properties of the first and second eigenfunction of this operator such as monotonicity and differentiability. Another main argument used in proving our spectral gap lower bound is some integral inequality which has important consequences in the embedding theory of Sobolev spaces of fractional order. This inequality is a consequence of the Garsia-Rodemich-Rumsey-Lemma (abbreviated as GRR-Lemma) [34]. The fact that this inequality follows from GRR-Lemma was observed by M. Kassmann in [39].

Our work is motivated by the classical results of M. Ashbaugh and R. Benguria obtained in [3, 4, where the similar spectral problem was studied for the classical Schrödinger operator with the symmetric single-well potential on the interval.

Before we describe our results in details let us recall the basic definitions and facts. Let $\left(X_{t}\right)_{t \geq 0}$ be the symmetric $\alpha$-stable process of order $\alpha \in(0,2)$ in $\mathbf{R}$. This process is a Markov process with stationary independent increments and the characteristic function of the form $\mathbf{E}^{0}\left[\exp \left(i \xi X_{t}\right)\right]=$ $\exp \left(-t|\xi|^{\alpha}\right), \xi \in \mathbf{R}, t>0$. As usual, $\mathbf{E}^{x}$ denotes the expected value of the process starting at $x \in \mathbf{R}$. Let $(a, b) \subset \mathbf{R},-\infty<a<b<\infty$, be an open interval and let $\tau_{(a, b)}=\inf \left\{t \geq 0: X_{t} \notin(a, b)\right\}$ be the first exit time of $X_{t}$ from $(a, b)$.

The Feynman-Kac semigroup $\left(T_{t}\right)_{t \geq 0}$ for the symmetric $\alpha$-stable process $X_{t}$ killed upon leaving $(a, b)$ and for potential $V \in \mathcal{V}^{\alpha}((a, b))$ is defined as

$$
T_{t} f(x)=\mathbf{E}^{x}\left[\exp \left(-\int_{0}^{t} V\left(X_{s}\right) d s\right) f\left(X_{t}\right) ; \tau_{(a, b)}>t\right], \quad f \in L^{2}((a, b)), t>0, x \in(a, b),
$$

where $\mathcal{V}^{\alpha}((a, b))$ is a class of functions $V:(a, b) \rightarrow \mathbf{R}$ specified by the following three conditions:

(i) integrability: $V$ extended to $\mathbf{R}$ by putting 0 outside $(a, b)$ is in the Kato class $\mathcal{K}^{\alpha}$ for the symmetric $\alpha$-stable process $X_{t}$. Formal definition of $\mathcal{K}^{\alpha}$ is given in Section 2.

(ii) symmetry: $V(x)=V(b+a-x)$ for $x \in(a, b)$.

Research supported by the Polish Ministry of Science and Higher Education grant no. N N201 527338. 
(iii) differentiability and monotonicity: $V^{\prime}$ exists in $(a, b)$ and $V^{\prime}(x) \leq 0$ for $x \in(a,(a+b) / 2)$. In the above definition we use the convention that potentials $V$ are defined on the interval $(a, b)$. However, very often, it will be useful to see the potential $V$ as a function extended to whole real line $\mathbf{R}$ by putting 0 outside $(a, b)$. Notice also that the assumption (i) is an integrability condition under which the above Feynman-Kac semigroup is well defined (see [14, 15]). Moreover, it immediately follows from the assumptions (ii) and (iii) that $V$ is a symmetric function, which is continuous, bounded from below, nonincreasing in $(a,(a+b) / 2)$ and nondecreasing in $((a+b) / 2, b)$. In the terminology of [3] potentials with a such monotonicity property are called symmetric single-well. We refer to the potentials from the class $\mathcal{V}^{\alpha}((a, b))$ as the symmetric differentiable single-well potentials on the interval $(a, b)$.

The operators $T_{t}$ are symmetric and form a strongly continuous semigroup on $L^{2}((a, b))$. The infinitesimal generator $L$ of the semigroup $\left(T_{t}\right)_{t \geq 0}$ is defined formally by

$$
L f=\lim _{t \downarrow 0} \frac{T_{t} f-f}{t}
$$

for such $f \in L^{2}((a, b))$ for which this limit exists in $L^{2}((a, b))$. The set of all such functions is called the domain of $L$ and is denoted by $\mathcal{D}(L)$. Similarly, we define $L f(x)=\lim _{t \downarrow 0}\left(T_{t} f(x)-f(x)\right) / t$ for any $f \in C((a, b))$ and $x \in(a, b)$ for which the limit exists. It is easy to show that if $f \in C_{\mathrm{c}}^{\infty}((a, b))$, then $L f(x)$ is well defined and

$$
L f(x)=-(-\Delta)^{\alpha / 2} f(x)-V(x) f(x), \quad x \in(a, b),
$$

where $-(-\Delta)^{\alpha / 2}$ is the fractional Laplacian of order $\alpha$ (see [16, p. 11-13]). Note that the operator $(-\Delta)^{\alpha / 2}$ will not be used in any essential way in proving our eigenvalue gap estimates. We just want to emphasize the connection between the operators $L$ and $(-\Delta)^{\alpha / 2}$. By this correspondence, we refer to the operator $-L$ as the fractional Schrödinger operator with the potential $V$ on interval $(a, b)$.

In recent years Schrödinger operators based on non-local pseudodifferential operators have been intensively studied. One of the most well known result is the so-called Hardy-Lieb-Thirring inequality obtained in 2008 by R. Frank, E. Lieb and R. Seiringer [36], which is connected with the problem of the stability of matter [47]. In the last 30 years many results concerning the fractional Schrödinger operators and relativistic Schrödinger operators have been obtained [20, 35, 53, 22, 23, 24, 14, 15, 43, 37, 38, 49]. These results are about functional integration, structure of spectrum, conditional gauge theorem, estimates of eigenfunctions and intrinsic ultracontractivity. Most of these results are obtained by using the probabilistic and potential theoretic methods.

The fact that the interval $(a, b)$ is bounded implies that for any $t>0$ the operator $T_{t}$ maps $L^{2}((a, b))$ into $L^{\infty}((a, b))$. It follows from the general theory of semigroups that there exists an orthonormal basis of eigenfunctions $\left\{\varphi_{n}\right\}$ in $L^{2}((a, b))$ and corresponding sequence of eigenvalues

$$
\lambda_{1}<\lambda_{2} \leq \lambda_{3} \leq \ldots \rightarrow \infty
$$

satisfying

$$
\begin{aligned}
T_{t} \varphi_{n} & =e^{-\lambda_{n} t} \varphi_{n}, \\
L \varphi_{n} & =-\lambda_{n} \varphi_{n}, \quad n \geq 1 .
\end{aligned}
$$

We may and do choose the basis $\left\{\varphi_{n}\right\}$ so that $\varphi_{n}$ is either symmetric (i.e., $\varphi_{n}(x)=\varphi_{n}(a+b-x)$ for $x \in(a, b)$ ) or antisymmetric (i.e., $\varphi_{n}(x)=-\varphi_{n}(a+b-x)$ for $x \in(a, b)$ ). Moreover, each eigenfunction $\varphi_{n}$ is continuous and bounded and all $\lambda_{n}$ have finite multiplicities. Additionally, $\lambda_{1}$ is simple and the corresponding eigenfunction, the so-called ground state eigenfunction, can be assumed to be strictly positive on $(a, b)$. Our main concern in this paper is the difference $\lambda_{2}-\lambda_{1}>0$, which is called the spectral gap. All above defined objects depend on the stability 
parameter $\alpha \in(0,2)$, the interval $(a, b)$ and the potential $V \in \mathcal{V}^{\alpha}((a, b))$. However, for simplicity, we prefer to omit this dependence in our notation.

Let us point out that $(-\Delta)^{\alpha / 2} \varphi_{n}(x)$ is well defined for all $x \in(a, b), n \geq 1$, and we have

$$
-L \varphi_{n}(x)=(-\Delta)^{\alpha / 2} \varphi_{n}(x)+V(x) \varphi_{n}(x)=\lambda_{n} \varphi_{n}(x), \quad x \in(a, b) .
$$

By the fact that each $\varphi_{n}$ may be extended to whole $\mathbf{R}$ by putting 0 outside $(a, b)$, the spectral problem discussed in this paper can be seen as the eigenproblem for the fractional Schrödinger operator on the interval $(a, b)$ with Dirichlet exterior conditions (that is, outside the interval $(a, b))$.

Mentioned above spectral problem has been widely studied for classical Schrödinger operators $-\Delta+V$ acting on $L^{2}(D)$ with Dirichlet boundary conditions, where $D$ is a bounded domain in $\mathbf{R}^{d}$, $d \geq 1$. Motivated by problems in mathematical physics concerning the behaviour of free Boson gases, M. van den Berg [11] made the following conjecture. If $D \subset \mathbf{R}^{d}$ is convex such that $\operatorname{diam}(D)<\infty$ and $V$ is nonnegative convex potential in $D$, then

$$
\lambda_{2, D}^{V}-\lambda_{1, D}^{V}>\frac{3 \pi^{2}}{\operatorname{diam}(D)^{2}}
$$

where $\lambda_{1, D}^{V}$ and $\lambda_{2, D}^{V}$ are the first and the second eigenvalue of $-\Delta+V$ acting on $L^{2}(D)$ with Dirichlet boundary conditions. This problem has been widely studied by many authors [50, 52, 28, 10, 51, 48, 1. In particular, the strict inequality (2) was obtained in 2010 by B. Andrews and J. Clutterbuck [1]. Let us point out that the this conjecture for intervals on the real line and for arbitrary nonnegative convex potentials was proved earlier by R. Lavine [46].

The classical result which is the most related to our one was obtained by M. Ashbaugh and R. Benguria [3, 4]. They studied this problem in one dimension when a domain $D$ is just a bounded interval and showed the inequality (2) for the different class of symmetric single-well potentials $V$ that are integrable in $D \subset \mathbf{R}$. This class includes the symmetric convex potentials, as well as a variety of nonconvex (but symmetric) potentials.

The problem of eigenvalue estimates and the spectral gap lower bound has also been studied for fractional Laplacian $-(-\Delta)^{\alpha / 2}$ (i.e. $V \equiv 0$ ) on bounded domains of $\mathbf{R}^{d}$ with Dirichlet exterior conditions $[26,29,6,6,8,32,44,30$. In one dimension (when $D$ is an interval) eigenvalue gaps estimates follow from results in [5] $(\alpha=1)$ and [25] $(\alpha>1)$. Moreover, the recent papers [42] $(\alpha=1)$, 45] $(\alpha \in(0,2))$ contain new asymptotic formulas for eigenvalues, which can be used to find the numerical bounds for eigenvalue gaps.

Now we formulate the main results of this paper. The following variational formula for the gaps between eigenvalues of $L$ is a rather standard fact. In particular, this formula gives a variational representation for the spectral gap $\lambda_{2}-\lambda_{1}$ and will be a starting point of our proofs. In fact, it is a fractional extension of the classical variational formula for the eigenvalue gaps of the classical Schrödinger operators which can be found for example in [51. For the version of this formula for the fractional Laplacian (i.e. $V \equiv 0)$ we refer to [32]. Denote by $L^{2}\left((a, b), \varphi_{1}^{2}\right)$ the space of square-integrable functions on the interval $(a, b)$ with measure $\varphi_{1}^{2}(x) d x$.

Proposition 1. Assume that $\alpha \in(0,2)$. Let $V \in \mathcal{V}^{\alpha}((a, b)),-\infty<a<b<\infty$. Then for every $n \geq 2$ we have

$$
\lambda_{n}-\lambda_{1}=\inf _{f \in \mathcal{F}_{n}} \frac{\mathcal{A}_{-\alpha}}{2} \int_{a}^{b} \int_{a}^{b} \frac{(f(x)-f(y))^{2}}{|x-y|^{1+\alpha}} \varphi_{1}(x) \varphi_{1}(y) d x d y
$$

where

$$
\mathcal{F}_{n}=\left\{f \in L^{2}\left((a, b), \varphi_{1}^{2}\right): \int_{a}^{b} f^{2}(x) \varphi_{1}^{2}(x) d x=1, \int_{a}^{b} f(x) \varphi_{1}(x) \varphi_{i}(x) d x=0,1 \leq i \leq n-1\right\}
$$


and

$$
\mathcal{A}_{\gamma}=\frac{\Gamma((1-\gamma) / 2)}{2^{\gamma} \sqrt{\pi}|\Gamma(\gamma / 2)|} .
$$

Moreover, the infimum in (3) is achieved for $f=\varphi_{n} / \varphi_{1}$.

Proposition 1 is a consequence of the standard variational formula for eigenvalues and a special case of [21, Theorems 2.6 and 2.8]. Its proof is given at the end of Section 2.

Our first main results are the following theorems concerning the properties of eigenfunctions. The first one is about the monotonicity of $\varphi_{1}$. We extend some earlier ideas from [7, 9] and [10], and prove that the ground state eigenfunction is unimodal in the interval $(a, b)$. This property will be our main argument in proving eigenvalue gap estimates.

Theorem 1. Let $\alpha \in(0,2)$. Let $V \in \mathcal{V}^{\alpha}((a, b)),-\infty<a<b<\infty$. Then $\varphi_{1}$ is symmetric and unimodal in $(a, b)$, i.e., $\varphi_{1}$ is nondecreasing in $(a,(a+b) / 2)$ and nonincreasing in $((a+b) / 2, b)$.

Theorem 2. Let $\alpha \in(1,2)$. Let $V \in \mathcal{V}^{\alpha}((a, b)),-\infty<a<b<\infty$. Then for all $n \geq 1$ the derivative of $\varphi_{n}$ exists in $(a, b)$. Moreover, if $[c, d] \subset(a, b)$, then there exists a constant $C_{V, n, \alpha, a, b, c, d}$ such that for all $x \in[c, d]$ we have

$$
\left|\frac{d}{d x} \varphi_{n}(x)\right| \leq C_{V, n, \alpha, a, b, c, d}\left\|\varphi_{n}\right\|_{\infty} .
$$

Let us recall that our orthonormal basis $\left\{\varphi_{n}\right\}$ is chosen so that each $\varphi_{n}$ is either symmetric or antisymmetric in $(a, b)$. It follows from the fact that $\left\{\varphi_{n}\right\}$ is an orthonormal basis that among $\varphi_{n}$ there are infinitely many antisymmetric functions in $(a, b)$. Denote by $\lambda_{*}$ the smallest eigenvalue corresponding to the antisymmetric eigenfunction $\varphi_{*}$. It is a natural hypothesis that $\lambda_{*}=\lambda_{2}$. For the classical Schrödinger operator on the the interval this fact is well known. It is a consequence of the Courant-Hilbert theorem which states that $\varphi_{2}$ has exactly two nodal domains (the interval consists of exactly two subintervals on which the sign of $\varphi_{2}$ is fixed). In our case this problem is more complicated. This is due to the fact that no version of the Courant-Hilbert theorem is known for operators which are non-local. Despite this fact, this hypothesis was proved by R. Bañuelos and T. Kulczycki [6] for $\alpha=1$ and $V \equiv 0$. Very recently, M. Kwaśnicki proved this hypothesis in [45] for $\alpha \in(1,2)$ and $V \equiv 0$.

The following lower bound for $\lambda_{*}-\lambda_{1}$ is derived by using Theorem 1 only.

Theorem 3. Assume that $\alpha \in(0,2)$. Let $V \in \mathcal{V}^{\alpha}((a, b)),-\infty<a<b<\infty$. Let $\lambda_{*}$ be the smallest eigenvalue corresponding to the antisymmetric eigenfunction $\varphi_{*}$. Then we have

$$
\lambda_{*}-\lambda_{1} \geq \frac{\mathcal{A}_{-\alpha}}{(b-a)^{\alpha}} .
$$

The next theorem is the main result of this paper.

Theorem 4. Assume that $\alpha \in(1,2)$. Let $V \in \mathcal{V}^{\alpha}((a, b)),-\infty<a<b<\infty$. Then we have

$$
\lambda_{2}-\lambda_{1} \geq \frac{C_{\alpha}^{(3)}}{(b-a)^{\alpha}},
$$

where $C_{\alpha}^{(3)}=\frac{\mathcal{A}_{-\alpha}}{4}\left(\left(\frac{\alpha-1}{16(\alpha+1)}\right)^{2} \vee\left(\frac{\alpha-1}{12(\alpha+1)}\right)^{\alpha+1} \vee\left(\frac{1}{9}\right)^{\frac{\alpha+1}{\alpha-1}}\right)$

Since we do not know whether the second eigenfunction is antisymmetric, we also have to consider the case that it is symmetric in the proof of Theorem 4. Our crucial argument applied to the proof in this case is the following integral inequality which is known to have important consequences in the embedding theory of Sobolev spaces of fractional order. 
Proposition 2. Assume that $\alpha \in(1,2)$. Let $-\infty<a<b<\infty$ and let $f$ be a Lipschitz function in $[a, b]$. Then

$$
\int_{a}^{b} \int_{a}^{b} \frac{(f(x)-f(y))^{2}}{|x-y|^{1+\alpha}} d x d y \geq C_{\alpha}^{(4)} \frac{(f(b)-f(a))^{2}}{(b-a)^{\alpha-1}}
$$

with $C_{\alpha}^{(4)}=\left(\frac{\alpha-1}{16(\alpha+1)}\right)^{2} \vee\left(\frac{\alpha-1}{12(\alpha+1)}\right)^{\alpha+1} \vee\left(\frac{1}{3}\right)^{2 \frac{\alpha+1}{\alpha-1}}$.

In [39] M. Kassmann showed that the inequalities of the form (7) are the direct corollary from the GRR-type inequalities. The constant $((\alpha-1) /(16(\alpha+1)))^{2}$ is derived from the classical onedimensional GRR-lemma [34, Lemma 1.1], while the constant $((\alpha-1) /(12(\alpha+1)))^{\alpha+1}$ is a consequence of the Kassmann's proof [39, Proof of Theorem 3]. To obtain the inequality with this constant he used the multidimensional extension of GRR-Lemma by L. Arnold and P. Imkeller [2]. Note that there are several improvements of the GRR-lemma in various directions. However, according to the author's knowledge these two constants seem to be the best constants known up to now in the one-dimensional inequality (7). Since our main concern here is to obtain possibly the best constant in the inequality (7), we propose a completely different proof of the inequality (7) based on some inductive argument. This direct and much more self-contained procedure allows us to obtain the constant $3^{-2(\alpha+1) /(\alpha-1)}$ which is better for $\alpha$ near 2 (approximately for $\alpha>1.736$ ). We discuss the structure of the constant $C_{\alpha}^{(4)}$ in Section 5 (see Remark 2 and Figures 1 and 2 .

Remark 1. Let $1<\beta<p<\infty$. Let $-\infty<a<b<\infty$ and let $f$ be a continuous function in $[a, b]$. More general inequalities of the form

$$
\int_{a}^{b} \int_{a}^{b} \frac{(f(x)-f(y))^{p}}{|x-y|^{1+\beta}} d x d y \geq C_{\beta, p}^{(5)} \frac{(f(b)-f(a))^{p}}{(b-a)^{\beta-1}}
$$

and their multidimensional versions are known to be the direct corollaries from the GRR-type inequalities. Their direct consequence is that if for some function $f$ the double integral on the left hand side is finite, then the function $f$ is Hölder continuous with exponent $(\beta-1) / p$. As shown in [39, these inequalities directly imply the embedding theorems from the so-called Sobolev-Slobodecki spaces into the spaces of Hölder continuous functions with exponent smaller or equal to $(\beta-1) / p$ (for more details see [39] and references therein). Simple modification of the inductive part of our proof of Proposition 2 also gives the inequality (8) with constant $C_{\beta, p}^{(5)}=3^{-p(\beta+1) /(\beta-1)}$ for Lipschitz continuous functions.

The following counterexample shows that in the case $\alpha \in(0,1)$ the inequality $(7)$ does not hold with any positive constant. This is a reason why our method of the proof of bound (6) does not work in this case. The case $\alpha=1$ remains open also.

Example 1. Assume that $\alpha \in(0,1)$. Let $f$ be a $C^{\infty}$-class function such that

$$
f(x)= \begin{cases}0, & x<1 / 4, \\ \in[0,1), & 1 / 4 \leq x<1 / 2, \\ 1, & x \geq 1 / 2\end{cases}
$$

Consider the sequence of functions of the form $f_{n}(x)=f(n x), n \geq 1$. Clearly, each $f_{n}$ is a $C^{\infty}$-class function such that $f_{n}(0)=0, f_{n}(1)=1$. However,

$$
\int_{0}^{1} \int_{0}^{1} \frac{\left(f_{n}(x)-f_{n}(y)\right)^{2}}{|x-y|^{1+\alpha}} d x d y \rightarrow 0 \quad \text { as } n \rightarrow \infty .
$$

The justification of the above example is a very special version of similar one in [31, Section 2] and is given in Section 5 .

Note that the constants $C_{\alpha}^{(3)}$ and $C_{\alpha}^{(4)}$ obtained in Theorem 4 and Proposition 2 respectively, are not optimal. As we will see below, the inequality (7) is an important argument used in proving the 
bound (6). Indeed, we have $C_{\alpha}^{(3)}=\mathcal{A}_{-\alpha} / 4 C_{\alpha}^{(4)}$. It follows that by improving the constant $C_{\alpha}^{(4)}$ in the inequality (7), one can improve the constant $C_{\alpha}^{(3)}$ in (6). Notice also that in view of Theorem 3 another way to improve the constant in Theorem 4 is to show that $\lambda_{*}=\lambda_{2}$.

A consequence of Theorem 2 is the following fractional version of the weighted Poincaré inequality.

Corollary 1. Assume that $\alpha \in(1,2)$. Let $-\infty<a<b<\infty$ and let $g:[a, b] \rightarrow \mathbf{R}$ be continuous, nonincreasing and strictly positive in $[a, b)$. Let $f$ be a Lipschitz function on the interval $[a, b]$ such that $f(a)=0$. Then

$$
\int_{a}^{b} \int_{a}^{b} \frac{(f(x)-f(y))^{2}}{|x-y|^{1+\alpha}} g(x) g(y) d x d y \geq \frac{C_{\alpha}^{(4)}}{(b-a)^{\alpha}} \int_{a}^{b} f^{2}(x) g^{2}(x) d x,
$$

where $C_{\alpha}^{(4)}$ is given in Theorem 2.

The paper is organized as follows. In Section 2 we introduce additional notation and collect various facts which are used below. In particular, here we justify the variational formulas for eigenvalue gaps. In Section 3 we prove the properties of eigenfunctions. Section 4 contains the proof of Theorem 3. In Section 5 we show the crucial inequality in Proposition 2 and justify Example 1. Here we also discuss the structure of the constant $C_{\alpha}^{(4)}$ in Proposition 2. Section 6 contains the proof of main theorem concerning the spectral gap lower bound.

\section{Preliminaries}

Let $\alpha \in(0,2)$. By $C_{\alpha, \kappa}$ we always mean a strictly positive and finite constant depending on $\alpha$ and parameter $\kappa$. We adopt the convention that constants in some proofs may change their value from one use to another. However, very often, especially in the statements of our results, we write $C_{\kappa}^{(1)}, C_{\kappa}^{(2)}$ etc to distinguish between constants.

We now summarize the properties of the symmetric $\alpha$-stable process and some facts from its potential theory. For further information on the potential theory of stable processes we refer to [40, 23, 16, 19].

Let $X=\left(X_{t}\right)_{t \geq 0}$ be the standard one-dimensional symmetric $\alpha$-stable process with the Lévy measure $\nu(d x)=\mathcal{A}_{-\alpha}|x|^{-1-\alpha} d x$, where the constant $\mathcal{A}_{-\alpha}$ is given by (4). By $\mathbf{P}^{x}$ we denote the distribution of the process starting at $x \in \mathbf{R}$. For each fixed $t>0$ the transition density $p(t, y-x)$, $t>0, x, y \in \mathbf{R}$, of the process $X$ is a continuous and bounded function on $\mathbf{R} \times \mathbf{R}$ satisfying the following estimates

$$
C_{\alpha}^{-1}\left(\frac{t}{|y-x|^{1+\alpha}} \wedge t^{-1 / \alpha}\right) \leq p(t, y-x) \leq C_{\alpha}\left(\frac{t}{|y-x|^{1+\alpha}} \wedge t^{-1 / \alpha}\right) .
$$

Denote by

$$
q(t, y-x)=\frac{1}{\sqrt{4 \pi t}} \exp \left(-\frac{|x-y|^{2}}{4 t}\right), \quad t>0, x, y \in \mathbf{R},
$$

the transition density of the standard one dimensional Brownian motion $\left(B_{t}\right)_{t \geq 0}$ runing at twice the speed.

It is well known that the symmetric $\alpha$-stable process $\left(X_{t}\right)_{t \geq 0}$ can be represented as

$$
X_{t}=B_{\eta_{t}},
$$

where $\left(\eta_{t}\right)_{t \geq 0}$ is an $\alpha / 2$-stable subordinator independent of $\left(B_{t}\right)_{t \geq 0}$ (see [16]). Thus

$$
p(t, y-x)=\int_{0}^{\infty} q(s, y-x) g_{\alpha / 2}(t, s) d s,
$$

where $g_{\alpha / 2}(t, s)$ is the transition density of $\eta_{t}$. 
It is known that when $\alpha<1$, the process $X$ is transient with potential kernel [12]

$$
K^{(\alpha)}(y-x)=\int_{0}^{\infty} p(t, y-x) d t=\mathcal{A}_{\alpha}|y-x|^{\alpha-1}, \quad x, y \in \mathbf{R} .
$$

Whenever $\alpha \geq 1$ the process is recurrent (pointwise recurrent when $\alpha>1$ ). In this case we can consider the compensated kernel [13], that is, for $\alpha \geq 1$ we put

$$
K^{(\alpha)}(y-x)=\int_{0}^{\infty}\left(p(t, y-x)-p\left(t, x_{0}\right)\right) d t
$$

where $x_{0}=0$ for $\alpha>1$, and $x_{0}=1$ for $\alpha=1$. In this case

$$
K^{(\alpha)}(x)=\frac{1}{\pi} \log \frac{1}{|x|}
$$

for $\alpha=1$ and

$$
K^{(\alpha)}(x)=(2 \Gamma(\alpha) \cos (\pi \alpha / 2))^{-1}|x|^{\alpha-1}, \quad x \in \mathbf{R}
$$

for $\alpha>1$. Note that $K^{(\alpha)}(x) \leq 0$ if $\alpha>1$.

We say that the Borel function $V: \mathbf{R} \rightarrow \mathbf{R}$ belongs to the Kato class $\mathcal{K}^{\alpha}$ corresponding to the symmetric $\alpha$-stable process $X$ if $V$ satisfies either of the two equivalent conditions (see [53] and [15, $(2.5)])$

$$
\begin{gathered}
\limsup _{\epsilon \rightarrow 0} \int_{x \in \mathbf{R}} \int_{|y-x|<\epsilon}|V(y)|\left|K^{(\alpha)}(y-x)\right| d y=0, \\
\limsup _{t \rightarrow 0} \mathbf{E}_{x \in \mathbf{R}}^{x}\left[\int_{0}^{t}\left|V\left(X_{s}\right)\right| d s\right]=0 .
\end{gathered}
$$

For instance, if $V(x)=\left(1-x^{2}\right)^{-\beta}, \beta>0$, then $V \in \mathcal{K}^{\alpha}$ and $V \in \mathcal{V}^{\alpha}((-1,1))$ provided that $\beta<\alpha \wedge 1$. It can be verified directly that for every $\alpha \in(0,2), \mathcal{K}^{\alpha} \subset L_{\text {loc }}^{1}(\mathbf{R})$.

We denote by $p_{D}(t, x, y)$ the transition density of the process killed upon exiting an open bounded set $D \subset \mathbf{R}$. It satisfies the relation

$$
p_{D}(t, x, y)=p(t, y-x)-\mathbf{E}^{x}\left[p\left(t-\tau_{D}, y-X_{\tau_{D}}\right) ; \tau_{D} \leq t\right], \quad x, y \in D, t>0,
$$

where $\tau_{D}=\inf \left\{t>0: X_{t} \notin D\right\}$ is the first exit time from $D$. For every $t>0, x, y \in D$, we have

$$
0<p_{D}(t, x, y) \leq p(t, y-x)
$$

Let us recall that the semigroup of the process killed upon leaving set $D$ is given by

$$
P_{t}^{D} f(x)=\mathbf{E}^{x}\left[f\left(X_{t}\right) ; \tau_{D}>t\right]=\int_{D} f(y) p_{D}(t, x, y) d y, \quad f \in L^{2}(D), x \in D .
$$

The Green operator of an open bounded set $D$ is denoted by $G_{D}$. We set

$$
G_{D}(x, y)=\int_{0}^{\infty} p_{D}(t, x, y) d t
$$

and call $G_{D}(x, y)$ the Green function for $D$. We have

$$
G_{D} f(x)=\mathbf{E}^{x}\left[\int_{0}^{\tau_{D}} f\left(X_{t}\right) d t\right]=\int_{D} G_{D}(x, y) f(y) d y,
$$

for non-negative Borel function $f$ on $\mathbf{R}$.

We now discuss some selected properties of the Feynman-Kac semigroup for the fractional Schrödinger operator with a potential $V$ on bounded intervals of $\mathbf{R}$, which are needed below. For the rest of this section we assume that $D$ is a bounded interval $(a, b) \subset \mathbf{R}, a<b$, and $V \in \mathcal{V}^{\alpha}((a, b))$. We refer the reader to [14, 15, 22, 23, 27] for more systematic treatment of fractional Schrödinger operators. 
The $V$-Green operator for $(a, b)$ is defined by

$$
G_{(a, b)}^{V} f(x)=\int_{0}^{\infty} T_{t} f(x) d t=\mathbf{E}^{x}\left[\int_{0}^{\tau_{(a, b)}} e^{-\int_{0}^{t} V\left(X_{s}\right) d s} f\left(X_{t}\right) d t\right],
$$

for non-negative Borel functions $f$ on $(a, b)$. The corresponding gauge function is given by (see e.g. [14, p. 58], [23, 27])

$$
u_{(a, b)}(x)=\mathbf{E}^{x}\left[e^{-\int_{0}^{\tau}(a, b)} V\left(X_{s}\right) d s\right], \quad x \in(a, b) .
$$

When it is bounded in $(a, b)$, then $((a, b), V)$ is said to be gaugeable. It is easy to check that if $V \geq 0$ on $(a, b)$, then gaugebility holds.

The following perturbation type formula will be an important argument in the proof of differentiability of eigenfunctions. For the potential $V$ such that $((a, b), V)$ is gaugable and for bounded function $f$ we have (see [14, Formula 9])

$$
G_{(a, b)}^{V} f(x)=G_{(a, b)} f(x)-G_{(a, b)}\left(V G_{(a, b)}^{V} f\right)(x), \quad x \in(a, b) .
$$

Since $V \in \mathcal{V}^{\alpha}((a, b))$, inf $V>-\infty$. Observe that when $V \equiv 0$, then $\left(T_{t}\right)_{t \geq 0}$ is an usual semigroup of the symmetric stable process killed upon leaving $(a, b)$. That is for each $t \geq 0$ we have $T_{t}=P_{t}^{(a, b)}$. Generally, for $f \geq 0$ we have

$$
T_{t} f(x) \leq e^{-\inf V t} P_{t}^{(a, b)} f(x), \quad t>0, \quad x \in(a, b) .
$$

Using this fact, 12, 15), and the Riesz-Thorin interpolation theorem, it can be shown that for each $t>0$ the operators $T_{t}: L^{p}((a, b)) \rightarrow L^{q}((a, b)), 1 \leq p \leq q \leq \infty$, are bounded. Denote by $\left\|T_{t}\right\|_{p, q}$ the norm of a such operators.

Recall that the class $\mathcal{V}^{\alpha}((a, b))$ contains the signed potentials $V$. So we do not exclude the case that the operators $T_{t}$ are not sub-Markovian. However, each operator $T_{t}$ can be transformed to be sub-Markovian by adding a constant to the potential $V$. Indeed, if $\inf V<0$, then we put $V_{0}=$ $V-\inf V$. Clearly, $V_{0} \geq 0$, and thus, the corresponding Feynman-Kac semigroup is sub-Markovian. Denote this semigroup by $\left(T_{t}^{0}\right)_{t \geq 0}$. It can be checked directly that its generator is the operator $L_{0}=L+\inf V$ with purely discrete spectrum of the form $\left\{-\lambda_{1}+\inf V,-\lambda_{2}+\inf V,-\lambda_{3}+\inf V, \ldots\right\}$. However, both operators $L$ and $L_{0}$ have the same eigenvalue gaps $\lambda_{n}-\lambda_{1}, n \geq 1$. We will use this translation invariance property of the eigenvalue gaps in the sequel.

We now justify the variational formula in Proposition 1.

Proof of Proposition 1. By using the remark on the translation invariance of the eigenvalue gaps, we may and do assume that $V \geq 0$ and that the corresponding Feynman-Kac semigroup $\left(T_{t}\right)_{t \geq 0}$ is sub-Markovian. For every $t>0$ define operators:

$$
\widetilde{T}_{t} f=e^{\lambda_{1} t} \varphi_{1}^{-1} T_{t}\left(\varphi_{1} f\right), \quad f \in L^{2}\left((a, b), \varphi_{1}^{2}\right) .
$$

It is easy to see that operators $\widetilde{T}_{t}, t>0$, form a semigroup of symmetric Markov operators on $L^{2}\left((a, b), \varphi_{1}^{2}\right)$ such that

$$
\widetilde{T}_{t}\left(\frac{\varphi_{n}}{\varphi_{1}}\right)=e^{-\left(\lambda_{n}-\lambda_{1}\right) t} \frac{\varphi_{n}}{\varphi_{1}}, \quad n \geq 1
$$

Let

$$
\widetilde{\mathcal{E}}(f, f)=\lim _{t \rightarrow 0^{+}} \frac{1}{t}\left(f-\widetilde{T}_{t} f, f\right)_{L^{2}\left((a, b), \varphi_{1}^{2}\right)}
$$

for $f \in L^{2}\left((a, b), \varphi_{1}^{2}\right)$. It is known that the form $\widetilde{\mathcal{E}}$ with its natural domain

$$
\mathcal{D}(\widetilde{\mathcal{E}})=\left\{f \in L^{2}\left((a, b), \varphi_{1}^{2}\right): \widetilde{\mathcal{E}}(f, f)<\infty\right\}
$$


is the Dirichlet form corresponding to the semigroup $\left(\widetilde{T}_{t}\right)_{t>0}$ [33, p. 23]. By the standard variational formula for eigenvalues we have

$$
\lambda_{n}-\lambda_{1}=\inf _{f \in \mathcal{F}_{n}} \widetilde{\mathcal{E}}(f, f), \quad n \geq 2,
$$

and the infimum is achieved for $f=\varphi_{n} / \varphi_{1}$. Thus to complete the proof of Proposition 1 it is enough to see that

$$
\widetilde{\mathcal{E}}(f, f)=\frac{\mathcal{A}_{-\alpha}}{2} \int_{a}^{b} \int_{a}^{b} \frac{(f(x)-f(y))^{2}}{|x-y|^{1+\alpha}} \varphi_{1}(x) \varphi_{1}(y) d x d y .
$$

However, the equality (18) is a special case of more general results in [21, Theorems 2.6 and 2.8].

\section{Properties of Eigenfunctions}

For our convenience we work with the symmetric interval $(-a, a), 0<a<\infty$, in the proof of Theorem 1. First we need the following auxiliary lemmas. Let us note that our arguments in the proofs of Lemma 1, Lemma 2 and Theorem 11 extend some earlier ideas from [7, 9] and [10].

Lemma 1. Assume that $\alpha \in(0,2)$. Let $0<a<\infty$ and let $V$ be a bounded, symmetric and continuous function on $(-a, a)$ such that $V^{\prime}(x)$ exists in $(-a, a)$ except for at most finite points and $V$ is nonincreasing in $(-a, 0)$. Let $n$ be a natural number and let $s_{i}, t_{i}, i=1, \ldots, n$, be arbitrary positive parameters. For $x \in[-a, a]$ we define

$$
\Phi_{n}\left(x ; s_{1}, \ldots, s_{n}, t_{1}, \ldots, t_{n}\right)=\int_{-a}^{a} \ldots \int_{-a}^{a} \exp \left(-\sum_{i=1}^{n} t_{i} V\left(x_{i}\right)\right) \prod_{i=1}^{n} q\left(s_{i}, x_{i}-x_{i-1}\right) d x_{1} \ldots d x_{n},
$$

where $x_{0}=x$ and $q$ is given by (13). Then for every $n \geq 1$ and parameters $s_{i}, t_{i}, i=1, \ldots, n$, $\Phi_{n}\left(x ; s_{1}, \ldots, s_{n}, t_{1}, \ldots, t_{n}\right)$ is nondecreasing on $(-a, 0)$ and nonincreasing on $(0, a)$ as a function of $x$.

Proof. First note that for every $n \geq 1$ and positive parameters $s_{i}, t_{i}, i=1, \ldots, n, \Phi_{n}\left(x ; s_{1}, \ldots, s_{n}, t_{1}, \ldots, t_{n}\right)$ is symmetric and positive on $[-a, a]$. Without loss of generality we assume that $a=1$. We use the induction. Integrating by parts and using the fact that $V$ is symmetric, we have

$$
\begin{aligned}
& \sqrt{4 \pi} s_{1} \frac{d}{d x} \Phi_{1}\left(x ; s_{1}, t_{1}\right)=-\int_{-1}^{1} e^{-t_{1} V(y)} \frac{\partial}{\partial y} e^{-\frac{(y-x)^{2}}{4 s_{1}}} d y \\
& =e^{-\frac{(1+x)^{2}}{4 s_{1}}} \lim _{y \rightarrow-1} e^{-t_{1} V(y)}-e^{-\frac{(1-x)^{2}}{4 s_{1}}} \lim _{y \rightarrow 1} e^{-t_{1} V(y)}+\int_{-1}^{1} e^{-\frac{(y-x)^{2}}{4 s_{1}}} \frac{d}{d y} e^{-t_{1} V(y)} d y \\
& \quad=\lim _{y \rightarrow 1} e^{-t_{1} V(y)}\left(e^{-\frac{(1+x)^{2}}{4 s_{1}}}-e^{-\frac{(1-x)^{2}}{4 s_{1}}}\right)+\int_{0}^{1}\left(e^{-\frac{(y-x)^{2}}{4 s_{1}}}-e^{-\frac{(y+x)^{2}}{4 s_{1}}}\right) \frac{d}{d y} e^{-t_{1} V(y)} d y .
\end{aligned}
$$

Since

$$
e^{-\frac{(y-x)^{2}}{4 s}} \geq e^{-\frac{(y+x)^{2}}{4 s}}, \quad x, y, s>0
$$

and

$$
\frac{d}{d y} e^{-t V(y)}=-t e^{-t V(y)} V^{\prime}(y) \leq 0, \quad t>0,
$$

for almost all $y \in(0,1)$, the expression on the right hand side of $(19)$ is nonpositive for all $x \in(0,1)$. Thus, for every $s_{1}, t_{1}>0$ the function $\Phi_{1}\left(x ; s_{1}, t_{1}\right)$ is nonincreasing on $(0,1)$.

Now suppose that for any positive parameters $s_{1}, \ldots s_{n-1}, t_{1}, \ldots, t_{n-1}$ the function $\Phi_{n-1}$ is nonincreasing on $(0,1)$, that is, $\frac{d}{d x} \Phi_{n-1}\left(x ; s_{1}, \ldots, s_{n-1}, t_{1}, \ldots, t_{n-1}\right) \leq 0$ for $x \in(0,1)$. Our claim is 
$\frac{d}{d x} \Phi_{n}\left(x ; s_{1}, \ldots, s_{n}, t_{1}, \ldots, t_{n}\right) \leq 0$ for $x \in(0,1)$ and for every positive parameters $s_{1}, \ldots s_{n}, t_{1}, \ldots, t_{n}$. We observe that

$$
\Phi_{n}\left(x ; s_{1}, \ldots, s_{n}, t_{1}, \ldots, t_{n}\right)=\frac{1}{\sqrt{4 \pi s_{1}}} \int_{-1}^{1} e^{-\frac{(y-x)^{2}}{4 s_{1}}} e^{-t_{1} V(y)} \Phi_{n-1}\left(y ; s_{2}, \ldots, s_{n}, t_{2}, \ldots, t_{n}\right) d y .
$$

From now on, for more simplicity, we omit the parameters $s_{i}, t_{i}$ in our notation. Integrating by parts, similarly as before, we have

$$
\begin{aligned}
& \sqrt{4 \pi s_{1}} \frac{d}{d x} \Phi_{n}(x)=-\int_{-1}^{1} \frac{\partial}{\partial y}\left(e^{-\frac{(y-x)^{2}}{4 s_{1}}}\right) e^{-t_{1} V(y)} \Phi_{n-1}(y) d y \\
& \quad=\Phi_{n-1}(1) \lim _{y \rightarrow 1} e^{-t_{1} V(y)}\left(e^{-\frac{(1+x)^{2}}{4 s_{1}}}-e^{-\frac{(1-x)^{2}}{4 s_{1}}}\right)+\int_{-1}^{1} e^{-\frac{(y-x)^{2}}{4 s_{1}}} \frac{d}{d y}\left(e^{-t_{1} V(y)} \Phi_{n-1}(y)\right) d y .
\end{aligned}
$$

Observe that by (20) the first term on the right hand side of (21) is nonpositive. Thus it is enough to show that for all $x \in(0,1)$ the last integral also is nonpositive. Denote $h(y)=e^{-t_{1} V(y)} \Phi_{n-1}(y)$. Recall that $V^{\prime}(y) \geq 0$ for almost all $y \in(0,1)$. By induction hypothesis and by symmetry, we have respectively

$$
h^{\prime}(y)=\frac{d}{d y}\left(e^{-t_{1} V(y)} \Phi_{n-1}(y)\right)=-t_{1} V^{\prime}(y) e^{-t_{1} V(y)} \Phi_{n-1}(y)+e^{-t_{1} V(y)} \frac{d}{d y} \Phi_{n-1}(y) \leq 0
$$

and

$$
h^{\prime}(y)=-h^{\prime}(-y)
$$

for almost all $y \in(0,1)$. This and $(20)$ give for all $x \in(0,1)$, almost all $y \in(0,1)$ and $t_{1}, s_{1}>0$

$$
e^{-\frac{(y-x)^{2}}{4 s_{1}}} h^{\prime}(y)+e^{-\frac{(y+x)^{2}}{4 s_{1}}} h^{\prime}(-y)=e^{-\frac{(y-x)^{2}}{4 s_{1}}} h^{\prime}(y)-e^{-\frac{(y+x)^{2}}{4 s_{1}}} h^{\prime}(y)=\left(e^{-\frac{(y-x)^{2}}{4 s_{1}}}-e^{-\frac{(y+x)^{2}}{4 s_{1}}}\right) h^{\prime}(y) \leq 0 .
$$

Integrating this inequality on $[0,1]$, we get

$$
\int_{-1}^{1} e^{-\frac{(y-x)^{2}}{4 s_{1}}} \frac{d}{d y}\left(e^{-t_{1} V(y)} \Phi_{n-1}(y)\right) d y \leq 0,
$$

for $x \in(0,1)$, which ends the proof.

Lemma 2. Assume that $\alpha \in(0,2)$. Let $0<a<\infty$ and $V \in \mathcal{V}^{\alpha}((-a, a))$. For $t>0$ denote

$$
g_{t}(x)=\mathbf{E}^{x}\left[\exp \left(-\int_{0}^{t} V\left(X_{s}\right) d s\right) ; t<\tau_{(-a, a)}\right], \quad x \in(-a, a) .
$$

Then for every fixed $t>0$ the functions $g_{t}(x)$ is nondecreasing in $(-a, 0)$ and nonincreasing in $(0, a)$.

Proof. Let $V_{k}(x)=V(x) \wedge k, x \in(-a, a), k \geq 1$. First note that for every $x \in(-a, a)$ and $t>0$

$$
\int_{0}^{t} V_{k}\left(X_{s}\right) d s \rightarrow \int_{0}^{t} V\left(X_{s}\right) d s
$$

as $k \rightarrow \infty, \mathbf{P}^{x}$-almost surely, by the monotone convergence theorem. Thus for all $x \in(-a, a)$ and $t>0$ we have

$$
\mathbf{E}^{x}\left[\exp \left(-\int_{0}^{t} V\left(X_{s}\right) d s\right) ; t<\tau_{(-a, a)}\right]=\lim _{k \rightarrow \infty} \mathbf{E}^{x}\left[\exp \left(-\int_{0}^{t} V_{k}\left(X_{s}\right) d s\right) ; t<\tau_{(-a, a)}\right]
$$

as a consequence of the fact that

$$
\exp \left(-\int_{0}^{t} V_{k}\left(X_{s}\right) d s\right) \leq \exp (-(\inf V \wedge 1) t), \quad t>0, \quad k \geq 1,
$$


and the bounded convergence theorem. Thus, it suffices to show that for any $k \geq 1$

$$
\mathbf{E}^{x}\left[\exp \left(-\int_{0}^{t} V_{k}\left(X_{s}\right) d s\right) ; t<\tau_{(-a, a)}\right]
$$

is nondecreasing in $(-a, 0)$ and nonincreasing in $(0, a)$ as a function of variable $x$, for each fixed $t>0$.

Fix now $k \geq 1$. By using the fact that $\mathbf{P}^{x}\left(X_{\tau_{(-a, a)}} \in\{-a, a\}\right)=0$ for $x \in(-a, a)$ and the paths are càdlàg, the boundedness and continuity of $V_{k}$, and the Markov property of the process $\left(X_{t}\right)_{t \geq 0}$, we have

$$
\begin{aligned}
\mathbf{E}^{x} & {\left[\exp \left(-\int_{0}^{t} V_{k}\left(X_{s}\right) d s\right) ; t<\tau_{(-a, a)}\right] } \\
& =\mathbf{E}^{x}\left[\exp \left(-\int_{0}^{t} V_{k}\left(X_{s}\right) d s\right) ; X_{s} \in(-a, a), \forall 0 \leq s \leq t\right] \\
& =\lim _{n \rightarrow \infty} \mathbf{E}^{x}\left[\exp \left(-\frac{t}{2^{n}} \sum_{i=1}^{2^{n}} V_{k}\left(X_{i t / 2^{n}}\right)\right) ; X_{i t / 2^{n}} \in(-a, a), i=1,2, \ldots, 2^{n}\right] \\
& =\lim _{n \rightarrow \infty} \int_{-a}^{a} \ldots \int_{-a}^{a} \prod_{i=1}^{2^{n}} \exp \left(-\frac{t}{2^{n}} V_{k}\left(x_{i}\right)\right) p\left(t / 2^{n}, x_{i}-x_{i-1}\right) d x_{1} \ldots d x_{2^{n}},
\end{aligned}
$$

where $x_{0}=x$. Moreover, by the subordination formula (14) and Fubini's theorem, the last multiple integral can be rewritten as

$$
\begin{aligned}
& \int_{-\infty}^{\infty} \ldots \int_{-\infty}^{\infty}\left(\int_{-a}^{a} \ldots \int_{-a}^{a} \prod_{i=1}^{2^{n}} \exp \left(-\frac{t}{2^{n}} V_{k}\left(x_{i}\right)\right) q\left(s_{i}, x_{i}-x_{i-1}\right) d x_{1} \ldots d x_{2^{n}}\right) \\
& \times \prod_{i=1}^{2^{n}} g_{\alpha / 2}\left(t / 2^{n}, s_{i}\right) d s_{1} \ldots d s_{2^{n}} \\
& =\int_{-\infty}^{\infty} \ldots \int_{-\infty}^{\infty} \Phi_{2^{n}}\left(x ; s_{1}, \ldots, s_{2^{n}}, t / 2^{n}, \ldots, t / 2^{n}\right) \prod_{i=1}^{2^{n}} g_{\alpha / 2}\left(t / 2^{n}, s_{i}\right) d s_{1} \ldots d s_{2^{n}}
\end{aligned}
$$

where

$$
\Phi_{2^{n}}\left(x ; s_{1}, \ldots, s_{2^{n}}, t_{1}, \ldots, t_{2^{n}}\right):=\int_{-a}^{a} \ldots \int_{-a}^{a} \prod_{i=1}^{2^{n}} \exp \left(-t_{i} V_{k}\left(x_{i}\right)\right) q\left(s_{i}, x_{i}-x_{i-1}\right) d x_{1} \ldots d x_{2^{n}}
$$

with $x_{0}=x$. By Lemma 1 we obtain that for every natural $n$ and for any positive parameters $t_{i}, s_{i}$, $i=1, \ldots, 2^{n}$, the function $\Phi_{2^{n}}$ is nondecreasing in $(-a, 0)$ and nonincreasing in $(0, a)$. Letting now $n \rightarrow \infty$ in 22, we conclude that the same is true for $\mathbf{E}^{x}\left[\exp \left(-\int_{0}^{t} V_{k}\left(X_{s}\right) d s\right) ; t<\tau_{(-a, a)}\right]$, for any $k \geq 1$ and for each fixed $t>0$. Thus the proof is complete.

Proof of Theorem 1. Let $0<a<\infty$ and $V \in \mathcal{V}^{\alpha}((-a, a))$ be fixed. First we show a symmetry. Recall that $\varphi_{1}$ is strictly positive in $(-a, a)$ and suppose contrary that $\varphi_{1}$ is not symmetric. Thus $\widehat{\varphi}_{1}(x):=\varphi_{1}(x)+\varphi_{1}(-x)$ is also an eigenfunction of $-L$ corresponding to the eigenvalue $\lambda_{1}$ such that $\widehat{\varphi}_{1} \notin \operatorname{span}\left(\varphi_{1}\right)$. This gives a contradiction, because $\lambda_{1}$ has muliplicity one. Thus $\varphi_{1}$ is symmetric in $(-a, a)$. 
Let now $P_{\varphi_{1}}: L^{2}((-a, a)) \rightarrow L^{2}((-a, a))$ be the projection onto $\operatorname{span}\left(\varphi_{1}\right)$. By the fact that $e^{\lambda_{1} t} T_{t} P_{\varphi_{1}}=P_{\varphi_{1}} e^{\lambda_{1} t} T_{t}=P_{\varphi_{1}}$, we have for $t>2$

$$
\begin{aligned}
\sup _{x \in(-a, a)}\left|\left(e^{\lambda_{1} t} T_{t}-P_{\varphi_{1}}\right) \mathbf{1}_{(-a, a)}(x)\right| & \leq 2 a\left\|e^{\lambda_{1} t} T_{t}-P_{\varphi_{1}}\right\|_{1, \infty} \\
& \leq 2 a e^{2 \lambda_{1}}\left\|T_{1}\right\|_{2, \infty}\left\|e^{\lambda_{1}(t-2)} T_{t-2}-P_{\varphi_{1}}\right\|_{2}\left\|T_{1}\right\|_{1,2} .
\end{aligned}
$$

Since by the spectral theorem

$$
\left\|e^{\lambda_{1} t} T_{t}-P_{\varphi_{1}}\right\|_{2} \leq e^{-\left(\lambda_{2}-\lambda_{1}\right) t}
$$

we obtain

$$
\varphi_{1}(x)=\lim _{t \rightarrow \infty} C_{V} e^{\lambda_{1} t} T_{t} \mathbf{1}_{(-a, a)}(x)=\lim _{t \rightarrow \infty} C_{V} e^{\lambda_{1} t} \mathbf{E}^{x}\left[\exp \left(-\int_{0}^{t} V\left(X_{s}\right) d s\right) ; t<\tau_{(-a, a)}\right],
$$

uniformly in $x \in(-a, a)$, with $C_{V}=\left(\left\|\varphi_{1}\right\|_{1}\right)^{-1}$. Now the assertion of the theorem follows from Lemma 2 .

We need the following auxiliary lemma. It is a version of [18, Lemma 5.2] and [17, Lemma 10].

Lemma 3. Let $\alpha \in(1,2)$. Let $-\infty<a<b<\infty$ and let $f \in L^{1}((a, b))$ be a function such that for every interval $[c, d] \subset(a, b)$ we have $\sup _{x \in[c, d]}|f(x)|<\infty$. Then

$$
\frac{d}{d x} G_{(a, b)} f(x)=\int_{a}^{b} \frac{\partial}{\partial x} G_{(a, b)}(x, y) f(y) d y, \quad x \in(a, b),
$$

and for every interval $[c, d] \subset(a, b)$ there is a constant $C_{\alpha, f, a, b, c, d}<\infty$ such that

$$
\left|\frac{d}{d x} G_{(a, b)} f(x)\right| \leq C_{\alpha, f, a, b, c, d}, \quad x \in[c, d] .
$$

Proof. Recall that $\alpha \in(1,2)$. By [18, Formula (5)] we have

$$
G_{(a, b)}(x, y)=K^{(\alpha)}(x-y)-H(x, y), \quad x, y \in(a, b), x \neq y,
$$

where $K^{(\alpha)}(x-y)=(2 \Gamma(\alpha) \cos (\pi \alpha / 2))^{-1}|x-y|^{\alpha-1}$ and $H(x, y)=\mathbf{E}^{x}\left[K^{(\alpha)}\left(X_{\tau_{(a, b)}}-y\right)\right]$. In view of this equality we have

$$
\begin{aligned}
\frac{d}{d x} G_{(a, b)} f(x)= & \lim _{h \rightarrow 0} \int_{a}^{b} \frac{K^{(\alpha)}(x+h-y)-K^{(\alpha)}(x-y)}{h} f(y) d y \\
& -\lim _{h \rightarrow 0} \int_{a}^{b} \frac{H(x+h, y)-H(x, y)}{h} f(y) d y, \quad x \in(a, b) .
\end{aligned}
$$

First notice that both partial derivatives $\frac{\partial}{\partial x} K^{(\alpha)}(x-y), x \neq y$, and $\frac{\partial}{\partial x} H(x, y)$ exist (see (10) in [18]). For $x \in(a, b)$ denote $\delta(x)=(b-x) \wedge(x-a)$. From [18, Lemma 3.2] we have $\left|\frac{\partial}{\partial x} H(x, y)\right| \leq$ $C_{\alpha, a, b} \delta(x)^{-1}$. It follows that

$$
\begin{aligned}
\left|\frac{\partial}{\partial x} G_{(a, b)}(x, y)\right| & \leq\left|\frac{\partial}{\partial x} K^{(\alpha)}(x-y)\right|+\left|\frac{\partial}{\partial x} H(x, y)\right| \\
& \leq C_{\alpha}|x-y|^{\alpha-2}+C_{\alpha, a, b} \delta(x)^{-1}, \quad x, y \in(a, b), x \neq y .
\end{aligned}
$$

This inequality and properties of $f$ imply that for each fixed $x \in(a, b)$ the integral on the right hand side of $(23)$ is absolutely convergent. Thus, to obtain $(23)$ it is enough to show that

$$
\lim _{h \rightarrow 0} \int_{a}^{b}\left|F_{h}^{(1)}(x, y)\right||f(y)| d y+\lim _{h \rightarrow 0} \int_{a}^{b}\left|F_{h}^{(2)}(x, y)\right||f(y)| d y=0, \quad x \in(a, b),
$$


where

$$
\begin{gathered}
F_{h}^{(1)}(x, y)=\frac{K^{(\alpha)}(x+h-y)-K^{(\alpha)}(x-y)}{h}-\frac{\partial}{\partial x} K^{(\alpha)}(x-y), \\
F_{h}^{(2)}(x, y)=\frac{H(x+h, y)-H(x, y)}{h}-\frac{\partial}{\partial x} H(x, y) .
\end{gathered}
$$

Fix now $x \in(a, b)$. Let $|h|<\delta(x) / 4$. From [18, Lemma 3.2] and Lagrange's theorem we obtain

$$
\left|F_{h}^{(2)}(x, y)\right| \leq C_{\alpha, a, b} \delta(x)^{-1} .
$$

This estimate and the fact that $f \in L^{1}((a, b))$ give that

$$
\lim _{h \rightarrow 0} \int_{a}^{b}\left|F_{h}^{(2)}(x, y)\right||f(y)| d y=0
$$

by the dominated convergence theorem.

It suffices to show that

$$
\lim _{h \rightarrow 0} \int_{a}^{b}\left|F_{h}^{(1)}(x, y)\right||f(y)| d y=0
$$

for each fixed $x \in(a, b)$. Let $\beta \in(0,1 / 2)$ and

$$
\begin{aligned}
\int_{a}^{b}\left|F_{h}^{(1)}(x, y)\right||f(y)| d y= & \int_{(x-\beta \delta(x), x+\beta \delta(x))}\left|F_{h}^{(1)}(x, y)\right||f(y)| d y \\
& +\int_{(a, b) \cap(x-\beta \delta(x), x+\beta \delta(x))^{c}}\left|F_{h}^{(1)}(x, y)\right||f(y)| d y .
\end{aligned}
$$

Fix $x \in(a, b)$ and $\varepsilon>0$. We will show that for sufficiently small $|h|$ the left hand side of $(29)$ is smaller than $\varepsilon$. Let $[c, d] \subset(a, b)$ be such that $x \in(c, d)$. Denote $M=\sup _{x \in[c, d]}|f(x)|$. Let $\beta$ be small enough so that $(x-\beta \delta(x), x+\beta \delta(x)) \subset[c, d]$. It is known (see [18, proof of Lemma 5.2]) that

$$
\left|F_{h}^{(1)}(x, y)\right| \leq C_{\alpha}\left(|x+h-y|^{\alpha-2} \vee|x-y|^{\alpha-2}\right), \quad y \in(a, b), y \neq x, y \neq x+h .
$$

Hence for any $h \in \mathbf{R}$

$$
\begin{aligned}
\int_{x-\beta \delta(x)}^{x+\beta \delta(x)}\left|F_{h}^{(1)}(x, y)\right||f(y)| d y & \leq M C_{\alpha} \int_{x-\beta \delta(x)}^{x+\beta \delta(x)}\left(|x+h-y|^{\alpha-2}+|x-y|^{\alpha-2}\right) d y \\
& \leq 2 M C_{\alpha} \int_{-\beta \delta(x)}^{\beta \delta(x)}|y|^{\alpha-2} d y .
\end{aligned}
$$

It is clear that there exists $\beta$ small enough so that the above integral is smaller than $\varepsilon / 2$. Let us fix such $\beta$. Clearly, $F_{h}^{(1)}(x, y) \rightarrow 0$ as $h \rightarrow 0$ for any $x \neq y$. By 30

$$
\left|F_{h}^{(1)}(x, y)\right| \leq C_{\alpha}\left(2 \beta^{-1} \delta(x)^{-1} \vee 1\right),
$$

for $y \in(a, b) \cap(x-\beta \delta(x), x+\beta \delta(x))^{c}$ and $h \in(-\beta \delta(x) / 2, \beta \delta(x) / 2)$. Since $f \in L^{1}((a, b))$, the second integral on the right hand side of $(29)$ tends to 0 as $h$ tends to 0 by the bounded convergence theorem. Hence for $|h|$ sufficiently small the second integral on the right hand side of $[29)$ is smaller than $\varepsilon / 2$. This finishes the proof (28). Thus (23) is proved. The boundedness property (24) is a simple consequence of the estimate $(25)$ and the properties of $f$.

Proof of Theorem 2. Let $-\infty<a<b<\infty$ and $V \in \mathcal{V}^{\alpha}((a, b))$. The starting point of the proof are the eigenequations

$$
T_{t} \varphi_{n}=e^{-\lambda_{n} t} \varphi_{n}, \quad n \geq 1
$$


Since we do not exclude the case that $V$ is signed potential, it may happen that $\lambda_{n}<0$ for finitely many $n$. Put

$$
\eta=\left\{\begin{array}{lll}
0, & \text { if } & \inf V>0, \\
1, & \text { if } & \inf V=0, \\
-2 \inf V, & \text { if } & \inf V<0
\end{array}\right.
$$

Denote $V_{\eta}=V+\eta$. Then $V_{\eta}>0$ and $\left((a, b), V_{\eta}\right)$ is gaugeable (see p. 7). By (31) we clearly have

$$
e^{-\left(\lambda_{n}+\eta\right) t} \varphi_{n}(x)=e^{-\eta t} T_{t} \varphi_{n}(x)=\mathbf{E}^{x}\left[e^{-\int_{0}^{t} V_{\eta}\left(X_{s}\right) d s} \varphi_{n}\left(X_{t}\right) ; \tau_{(a, b)}>t\right], \quad x \in(a, b) .
$$

Using the fact that $\lambda_{n}+\eta>0, n \geq 1$, and integrating over $t$ the above equations we obtain

$$
\varphi_{n}(x)=\left(\lambda_{n}+\eta\right) G_{(a, b)}^{V_{\eta}} \varphi_{n}(x), \quad x \in(a, b), n \geq 1 .
$$

Applying now the perturbation formula (16) to this equality we get

$$
\varphi_{n}(x)=\left(\lambda_{n}+\eta\right) G_{(a, b)} \varphi_{n}(x)-G_{(a, b)}\left(V_{\eta} \varphi_{n}\right)(x), \quad x \in(a, b), n \geq 1,
$$

which can be rewritten as

$$
\varphi_{n}(x)=\left(\lambda_{n}+\eta\right) \int_{a}^{b} G_{(a, b)}(x, y) \varphi_{n}(y) d y-\int_{a}^{b} G_{(a, b)}(x, y) V_{\eta}(y) \varphi_{n}(y) d y .
$$

Since $\left\|\varphi_{n}\right\|_{\infty}<\infty, V_{\eta} \in L^{1}((a, b))$ and is continuous in $(a, b)$, the assumptions of Lemma 3 are satisfied. Thus for $x \in(a, b)$ we have

$$
\frac{d}{d x} \varphi_{n}(x)=\left(\lambda_{n}+\eta\right) \int_{a}^{b} \frac{\partial}{\partial x} G_{(a, b)}(x, y) \varphi_{n}(y) d y-\int_{a}^{b} \frac{\partial}{\partial x} G_{(a, b)}(x, y) V_{\eta}(y) \varphi_{n}(y) d y .
$$

A direct consequence of Lemma 3 is that also for any interval $[c, d] \subset(a, b)$ there is a constant $C_{V, \alpha, n, a, b, c, d}$ such that for all $x \in[c, d]$ we have

$$
\left|\frac{d}{d x} \varphi_{n}(x)\right| \leq C_{V, \alpha, n, a, b, c, d}
$$

\section{LOWER BOUND FOR $\lambda_{*}-\lambda_{1}$}

Proof of Theorem 3. Let $0<a<\infty$. With no loss of generality we provide the arguments for the symmetric interval $(-a, a)$ only. Let $V \in \mathcal{V}^{\alpha}((-a, a))$. Recall that our orthonormal basis $\left\{\varphi_{n}\right\}$ is chosen so that $\varphi_{n}$ are either symmetric or antisymmetric. Let $n_{0}$ be the smallest natural number such that $\varphi_{n_{0}}$ is antisymmetric in $(-a, a)$. Thus $\varphi_{*}=\varphi_{n_{0}}$. Let $f=\varphi_{*} / \varphi_{1}=\varphi_{n_{0}} / \varphi_{1}$. For every $\varepsilon \in(0, a)$ we have

$$
\begin{aligned}
\int_{-a}^{a} \int_{-a}^{a} \frac{(f(x)-f(y))^{2}}{|x-y|^{1+\alpha}} \varphi_{1}(x) \varphi_{1}(y) d x d y & \geq \int_{\varepsilon}^{a} \int_{\varepsilon}^{a} \frac{(f(x)-f(y))^{2}}{|x-y|^{1+\alpha}} \varphi_{1}(x) \varphi_{1}(y) d x d y \\
& +\int_{\varepsilon}^{a} \int_{-a}^{-\varepsilon} \frac{(f(x)-f(y))^{2}}{|x-y|^{1+\alpha}} \varphi_{1}(x) \varphi_{1}(y) d x d y \\
& +\int_{-a}^{-\varepsilon} \int_{-a}^{-\varepsilon} \frac{(f(x)-f(y))^{2}}{|x-y|^{1+\alpha}} \varphi_{1}(x) \varphi_{1}(y) d x d y \\
& +\int_{-a}^{-\varepsilon} \int_{\varepsilon}^{a} \frac{(f(x)-f(y))^{2}}{|x-y|^{1+\alpha}} \varphi_{1}(x) \varphi_{1}(y) d x d y
\end{aligned}
$$

Simple changes of variables in the last three integrals and the fact that $f$ is antisymmetric give that the last sum can be transformed to

$$
2 \int_{\varepsilon}^{a} \int_{\varepsilon}^{a}\left(\frac{(f(x)-f(y))^{2}}{|x-y|^{1+\alpha}}+\frac{(f(x)+f(y))^{2}}{(x+y)^{1+\alpha}}\right) \varphi_{1}(x) \varphi_{1}(y) d x d y .
$$


Clearly, this is bigger or equal to

$$
\begin{aligned}
2 \int_{\varepsilon}^{a} \int_{\varepsilon}^{a} \frac{(f(x)-f(y))^{2}+(f(x)+f(y))^{2}}{(x+y)^{1+\alpha}} & \varphi_{1}(x) \varphi_{1}(y) d x d y \\
& =4 \int_{\varepsilon}^{a} \int_{\varepsilon}^{a} \frac{f^{2}(x)+f^{2}(y)}{(x+y)^{1+\alpha}} \varphi_{1}(x) \varphi_{1}(y) d x d y,
\end{aligned}
$$

which, by symmetry, is equal to

$$
8 \int_{\varepsilon}^{a} \int_{\varepsilon}^{a} \frac{f^{2}(x)}{(x+y)^{1+\alpha}} \varphi_{1}(x) \varphi_{1}(y) d x d y .
$$

Thus, by Theorem 1, we have

$$
\begin{aligned}
\int_{-a}^{a} \int_{-a}^{a} \frac{(f(x)-f(y))^{2}}{|x-y|^{1+\alpha}} \varphi_{1}(x) \varphi_{1}(y) d x d y & \geq 8 \int_{\varepsilon}^{a} \int_{\varepsilon}^{a} \frac{f^{2}(x)}{(x+y)^{1+\alpha}} \varphi_{1}(x) \varphi_{1}(y) d y d x \\
& \geq 8 \int_{\varepsilon}^{a} \int_{\varepsilon}^{x} \frac{f^{2}(x)}{(x+y)^{1+\alpha}} \varphi_{1}(x) \varphi_{1}(y) d y d x \\
& \geq 8 \int_{\varepsilon}^{a} \int_{\varepsilon}^{x} d y \frac{f^{2}(x)}{(2 x)^{1+\alpha}} \varphi_{1}^{2}(x) d x \\
& \geq \frac{4}{(2 a)^{\alpha}} \int_{\varepsilon}^{a} \frac{x-\varepsilon}{x} f^{2}(x) \varphi_{1}^{2}(x) d x .
\end{aligned}
$$

Now, letting $\varepsilon \rightarrow 0$, we obtain

$$
\begin{aligned}
\int_{-a}^{a} \int_{-a}^{a} \frac{(f(x)-f(y))^{2}}{|x-y|^{1+\alpha}} & \varphi_{1}(x) \varphi_{1}(y) d x d y \\
& \geq \frac{4}{(2 a)^{\alpha}} \int_{0}^{a} f^{2}(x) \varphi_{1}^{2}(x) d x=\frac{2}{(2 a)^{\alpha}} \int_{-a}^{a} f^{2}(x) \varphi_{1}^{2}(x) d x=\frac{2}{(2 a)^{\alpha}} .
\end{aligned}
$$

Since $f=\varphi_{*} / \varphi_{1}$ is antisymmetric, the assertion of Theorem 3 follows simply from Proposition 1 .

\section{Crucial inequality}

Proof of Proposition 2. For more clarity we divide the proof into the two parts.

(Part 1) In this part we show that in fact it is enough to prove Proposition 2 for $a=0$ and $b=1$, i.e., for $[a, b]=[0,1]$. First note that if $f$ is a Lipschitz function on an arbitrary interval $[a, b]$, $-\infty<a<b<\infty$, then the function $\tilde{f}(x):=f((b-a) x+a)$ is a Lipschitz function in [0,1] such that $\tilde{f}(0)=f(a)$ and $\tilde{f}(1)=f(b)$. Suppose that the claimed inequality (7) is true for all Lipschitz functions on the interval $[0,1]$ with constant $C_{\alpha}^{(4)}$. Thus, by trivial change of variables, we have

$$
\begin{aligned}
\int_{a}^{b} \int_{a}^{b} \frac{(f(x)-f(y))^{2}}{|x-y|^{1+\alpha}} d x d y & =\int_{a}^{b} \int_{a}^{b} \frac{\left(\tilde{f}\left(\frac{x-a}{b-a}\right)-\tilde{f}\left(\frac{y-a}{b-a}\right)\right)^{2}}{|x-y|^{1+\alpha}} d x d y \\
& =\frac{1}{(b-a)^{\alpha-1}} \int_{0}^{1} \int_{0}^{1} \frac{(\tilde{f}(x)-\tilde{f}(y))^{2}}{|x-y|^{1+\alpha}} d x d y
\end{aligned}
$$

Since

$$
\int_{0}^{1} \int_{0}^{1} \frac{(\tilde{f}(x)-\tilde{f}(y))^{2}}{|x-y|^{1+\alpha}} d x d y \geq C_{\alpha}^{(4)}(\tilde{f}(1)-\tilde{f}(0))^{2}=C_{\alpha}^{(4)}(f(b)-f(a))^{2},
$$

the proof of Part 1 is complete.

(Part 2) In this part we prove the proposition for $a=0$ and $b=1$. By Part 1 this will give our result for arbitrary $-\infty<a<b<\infty$. First note that the constant $((\alpha-1) /(16(\alpha+1)))^{2}$ in the 
inequality (7) for $(a, b)=(0,1)$ can be obtained by taking $\psi(x)=|x|^{2}$ and $p(x)=|x|^{(\alpha+1) / 2}$ in the classical GRR-Lemma [34, Lemma 1.1.]. Indeed, from the inequality (1.2) in [34] we clearly get

$$
\begin{aligned}
|f(1)-f(0)| & \leq 16\left(\int_{0}^{1} \int_{0}^{1} \frac{(f(x)-f(y))^{2}}{|x-y|^{1+\alpha}} d x d y\right)^{\frac{1}{2}} \frac{\alpha+1}{2} \int_{0}^{1} u^{\frac{\alpha-1}{2}-1} d u \\
& \leq 16\left(\frac{\alpha+1}{\alpha-1}\right)\left(\int_{0}^{1} \int_{0}^{1} \frac{(f(x)-f(y))^{2}}{|x-y|^{1+\alpha}} d x d y\right)^{\frac{1}{2}} .
\end{aligned}
$$

The second constant $((\alpha-1) /(12(\alpha+1)))^{\alpha+1}$ was derived in a similar manner by M. Kassmann (see [39, Proof of Theorem 3]) from the multidimensional improvement of the GRR-Lemma in [2].

We now find the third constant of the form $9^{(-(\alpha+1) /(\alpha-1))}$ in the inequality (7). We use some new inductive argument, which is completely independent of the GRR-Lemma. If $f(0)=f(1)$, then the claimed inequality is trivial. Without loss of generality we may assume that $f(0)=1$ and $f(1)>0$. In fact, by the scaling property of the claimed inequality (7), we may and do assume that $f(1)=1$. Let $c<1 / 3$ be a constant. It will be chosen later. The rest of the proof is divided into the several steps.

(Step 1) Denote

$$
\left\{\begin{array}{l}
a_{1}=0 \\
b_{1}=1
\end{array}, \quad\left\{\begin{array}{l}
f_{a_{1}}=0 \\
f_{b_{1}}=1
\end{array}, \quad\left\{\begin{array}{l}
x_{1}=a_{1}+c \\
y_{1}=b_{1}-c
\end{array}, \quad\left\{\begin{array}{l}
f_{x_{1}}=f_{a_{1}}+\frac{1}{3} \\
f_{y_{1}}=f_{b_{1}}-\frac{1}{3}
\end{array},\right.\right.\right.\right.
$$

and define

$$
m_{1}=\min \left\{x \in\left(a_{1}, b_{1}\right): f(x)=f_{x_{1}}\right\} \quad \text { and } \quad M_{1}=\max \left\{x \in\left(a_{1}, b_{1}\right): f(x)=f_{y_{1}}\right\} .
$$

Clearly, $f_{y_{1}}-f_{x_{1}}=1 / 3$. If $m_{1} \notin\left(a_{1}, x_{1}\right)$ and $M_{1} \notin\left(y_{1}, b_{1}\right)$, then we have

$$
\begin{aligned}
\int_{0}^{1} \int_{0}^{1} \frac{(f(x)-f(y))^{2}}{|x-y|^{1+\alpha}} d x d y & \geq \int_{a_{1}}^{x_{1}} \int_{y_{1}}^{b_{1}} \frac{(f(x)-f(y))^{2}}{|x-y|^{1+\alpha}} d x d y \\
& \geq \frac{\left(f_{y_{1}}-f_{x_{1}}\right)^{2}\left(b_{1}-y_{1}\right)\left(x_{1}-a_{1}\right)}{\left|b_{1}-a_{1}\right|^{1+\alpha}}=\left(\frac{c}{3}\right)^{2} .
\end{aligned}
$$

If $m_{1} \in\left(a_{1}, x_{1}\right)$ or $M_{1} \in\left(y_{1}, b_{1}\right)$, we consider the next step.

(Step 2) If $m_{1} \in\left(a_{1}, x_{1}\right)$ let us take

$$
\left\{\begin{array}{l}
a_{2}=a_{1} \\
b_{2}=x_{1}
\end{array}, \quad\left\{\begin{array}{l}
f_{a_{2}}=f_{a_{1}} \\
f_{b_{2}}=f_{x_{1}}
\end{array}, \quad\left\{\begin{array}{l}
x_{2}=a_{2}+c^{2} \\
y_{2}=b_{2}-c^{2}
\end{array}, \quad\left\{\begin{array}{l}
f_{x_{2}}=f_{a_{2}}+\left(\frac{1}{3}\right)^{2} \\
f_{y_{2}}=f_{b_{2}}-\left(\frac{1}{3}\right)^{2}
\end{array} .\right.\right.\right.\right.
$$

If $m_{1} \notin\left(a_{1}, x_{1}\right)$ and $M_{1} \in\left(y_{1}, b_{1}\right)$ let us take

$$
\left\{\begin{array}{l}
a_{2}=y_{1} \\
b_{2}=b_{1}
\end{array}, \quad\left\{\begin{array}{l}
f_{a_{2}}=f_{y_{1}} \\
f_{b_{2}}=f_{b_{1}}
\end{array}, \quad\left\{\begin{array}{l}
x_{2}=a_{2}+c^{2} \\
y_{2}=b_{2}-c^{2}
\end{array}, \quad\left\{\begin{array}{l}
f_{x_{2}}=f_{a_{2}}+\left(\frac{1}{3}\right)^{2} \\
f_{y_{2}}=f_{b_{2}}-\left(\frac{1}{3}\right)^{2}
\end{array} .\right.\right.\right.\right.
$$

Define

$$
m_{2}=\min \left\{x \in\left(a_{2}, b_{2}\right): f(x)=f_{x_{2}}\right\} \quad \text { and } \quad M_{2}=\max \left\{x \in\left(a_{2}, b_{2}\right): f(x)=f_{y_{2}}\right\} .
$$

Clearly, $f_{y_{2}}-f_{x_{2}}=1 / 3^{2}, b_{2}-a_{2}=c$. When $m_{2} \notin\left(a_{2}, x_{2}\right)$ and $M_{2} \notin\left(y_{2}, b_{2}\right)$, we have

$$
\begin{aligned}
\int_{0}^{1} \int_{0}^{1} \frac{(f(x)-f(y))^{2}}{|x-y|^{1+\alpha}} d x d y & \geq \int_{a_{2}}^{x_{2}} \int_{y_{2}}^{b_{2}} \frac{(f(x)-f(y))^{2}}{|x-y|^{1+\alpha}} d x d y \\
& \geq \frac{\left(f_{y_{2}}-f_{x_{2}}\right)^{2}\left(b_{2}-y_{2}\right)\left(x_{2}-a_{2}\right)}{\left|b_{2}-a_{2}\right|^{1+\alpha}}=\frac{c^{4}}{3^{4} c^{1+\alpha}}=\left(\frac{c}{3}\right)^{2} \frac{1}{3^{2} c^{\alpha-1}} .
\end{aligned}
$$

If $m_{2} \in\left(a_{2}, x_{2}\right)$ or $M_{2} \in\left(y_{2}, b_{2}\right)$, we consider the next step. 
Suppose that after $n-1$ steps $m_{n-1} \in\left(a_{n-1}, x_{n-1}\right)$ or $M_{n-1} \in\left(y_{n-1}, b_{n-1}\right)$. Then let us consider the next step.

(Step $n$ ) If $m_{n-1} \in\left(a_{n-1}, x_{n-1}\right)$ let us take

$$
\left\{\begin{array}{l}
a_{n}=a_{n-1} \\
b_{n}=x_{n-1}
\end{array}, \quad\left\{\begin{array}{l}
f_{a_{n}}=f_{a_{n-1}} \\
f_{b_{n}}=f_{x_{n-1}}
\end{array}, \quad\left\{\begin{array}{l}
x_{n}=a_{n}+c^{n} \\
y_{n}=b_{n}-c^{n}
\end{array}, \quad\left\{\begin{array}{l}
f_{x_{n}}=f_{a_{n}}+\left(\frac{1}{3}\right)^{n} \\
f_{y_{n}}=f_{b_{n}}-\left(\frac{1}{3}\right)^{n}
\end{array} .\right.\right.\right.\right.
$$

If $m_{n-1} \notin\left(a_{n-1}, x_{n-1}\right)$ and $M_{n-1} \in\left(y_{n-1}, b_{n-1}\right)$ let us take

$$
\left\{\begin{array}{l}
a_{n}=y_{n-1} \\
b_{n}=b_{n-1}
\end{array}, \quad\left\{\begin{array}{l}
f_{a_{n}}=f_{y_{n-1}} \\
f_{b_{n}}=f_{b_{n-1}}
\end{array}, \quad\left\{\begin{array}{l}
x_{n}=a_{n}+c^{n} \\
y_{n}=b_{n}-c^{n}
\end{array}, \quad\left\{\begin{array}{l}
f_{x_{n}}=f_{a_{n}}+\left(\frac{1}{3}\right)^{n} \\
f_{y_{n}}=f_{b_{n}}-\left(\frac{1}{3}\right)^{n}
\end{array} .\right.\right.\right.\right.
$$

Define

$$
m_{n}=\min \left\{x \in\left(a_{n}, b_{n}\right): f(x)=f_{x_{n}}\right\} \quad \text { and } \quad M_{n}=\max \left\{x \in\left(a_{n}, b_{n}\right): f(x)=f_{y_{n}}\right\} .
$$

Of course, $f_{y_{n}}-f_{x_{n}}=1 / 3^{n}, b_{n}-a_{n}=c^{n-1}$. If $m_{n} \notin\left(a_{n}, x_{n}\right)$ and $M_{n} \notin\left(y_{n}, b_{n}\right)$, then we have

$$
\begin{aligned}
& \int_{0}^{1} \int_{0}^{1} \frac{(f(x)-f(y))^{2}}{|x-y|^{1+\alpha}} d x d y \geq \int_{a_{n}}^{x_{n}} \int_{y_{n}}^{b_{n}} \frac{(f(x)-f(y))^{2}}{|x-y|^{1+\alpha}} d x d y \\
& \geq \frac{\left(f_{y_{n}}-f_{x_{n}}\right)^{2}\left(b_{n}-y_{n}\right)\left(x_{n}-a_{n}\right)}{\left|b_{n}-a_{n}\right|^{1+\alpha}}=\frac{c^{2 n}}{3^{2 n} c^{(n-1)(1+\alpha)}}=\left(\frac{c}{3}\right)^{2}\left(\frac{1}{3^{2} c^{\alpha-1}}\right)^{n-1} .
\end{aligned}
$$

If $m_{n} \in\left(a_{n}, x_{n}\right)$ or $M_{n} \in\left(y_{n}, b_{n}\right)$, then we consider the $n+1$ step.

Recalling that $\alpha \in(1,2)$ and choosing $c$ to be $9^{\frac{-1}{\alpha-1}}$, we obtain that

$$
\left(\frac{1}{3^{2} c^{\alpha-1}}\right)^{n}=1 \quad \text { for all } n \geq 0
$$

It is enough to see that there exists $n_{0} \geq 1$ such that $m_{n_{0}} \notin\left(a_{n_{0}}, x_{n_{0}}\right)$ and $M_{n_{0}} \notin\left(y_{n_{0}}, b_{n_{0}}\right)$. Indeed, in this case we have

$$
\int_{0}^{1} \int_{0}^{1} \frac{(f(x)-f(y))^{2}}{|x-y|^{1+\alpha}} d x d y \geq\left(\frac{c}{3}\right)^{2}\left(\frac{1}{3^{2} c^{\alpha-1}}\right)^{n_{0}-1}=\left(\frac{c}{3}\right)^{2}=\left(\frac{1}{9}\right)^{\frac{\alpha+1}{\alpha-1}}
$$

Suppose contrary that for all $n \geq 1$ we have $m_{n} \in\left(a_{n}, x_{n}\right)$ or $M_{n} \in\left(y_{n}, b_{n}\right)$. This means that there exists a decreasing sequence of intervals $\left\{\left(a_{n}, b_{n}\right)\right\}_{n \geq 1}$ such that $\left|b_{n}-a_{n}\right|=\left(\frac{1}{9}\right)^{\frac{n-1}{\alpha-1}}$. By the fact that $f$ is a Lipschitz function on $[a, b]$, there is a constant $C$ such that

$$
\left(\frac{1}{3}\right)^{n}=\left|f\left(M_{n}\right)-f\left(m_{n}\right)\right| \leq C\left|M_{n}-m_{n}\right| \leq C\left|b_{n}-a_{n}\right|=C\left(\frac{1}{9}\right)^{\frac{n-1}{\alpha-1}}, \quad n \geq 1,
$$

which gives a contradiction. Thus the inequality (7) is true for $a=0$ and $b=1$ with constant $C_{\alpha}^{(4)}=\left(\frac{1}{9}\right)^{\frac{\alpha+1}{\alpha-1}}$, and the proof of the proposition is complete.

Remark 2. The three subintervals of the stability parameter $\alpha$ on which the constant $C_{\alpha}^{(4)}$ in Proposition 2 takes the three different forms can be estimated as follows: for $\alpha \in(1 ; 1.10492)$ we have $C_{\alpha}^{(4)}=\left(\frac{\alpha-1}{12(\alpha+1)}\right)^{\alpha+1}$, for $\alpha \in(1.10493 ; 1.73546)$ we have $C_{\alpha}^{(4)}=\left(\frac{\alpha-1}{16(\alpha+1)}\right)^{2}$, while for $\alpha \in(1.73547 ; 2)$ we obtain $C_{\alpha}^{(4)}=\left(\frac{1}{9}\right)^{\frac{\alpha+1}{\alpha-1}}$ (see Figures 1 and 2 ).

Justification of Example 1. We clearly have

$$
\int_{0}^{1} \int_{0}^{1} \frac{\left(f_{n}(x)-f_{n}(y)\right)^{2}}{|x-y|^{1+\alpha}} d x d y \leq \int_{0}^{1} \int_{0}^{\frac{1}{2 n}} \frac{\left(f_{n}(x)-f_{n}(y)\right)^{2}}{|x-y|^{1+\alpha}} d x d y+\int_{0}^{\frac{1}{2 n}} \int_{0}^{1} \frac{\left(f_{n}(x)-f_{n}(y)\right)^{2}}{|x-y|^{1+\alpha}} d x d y .
$$




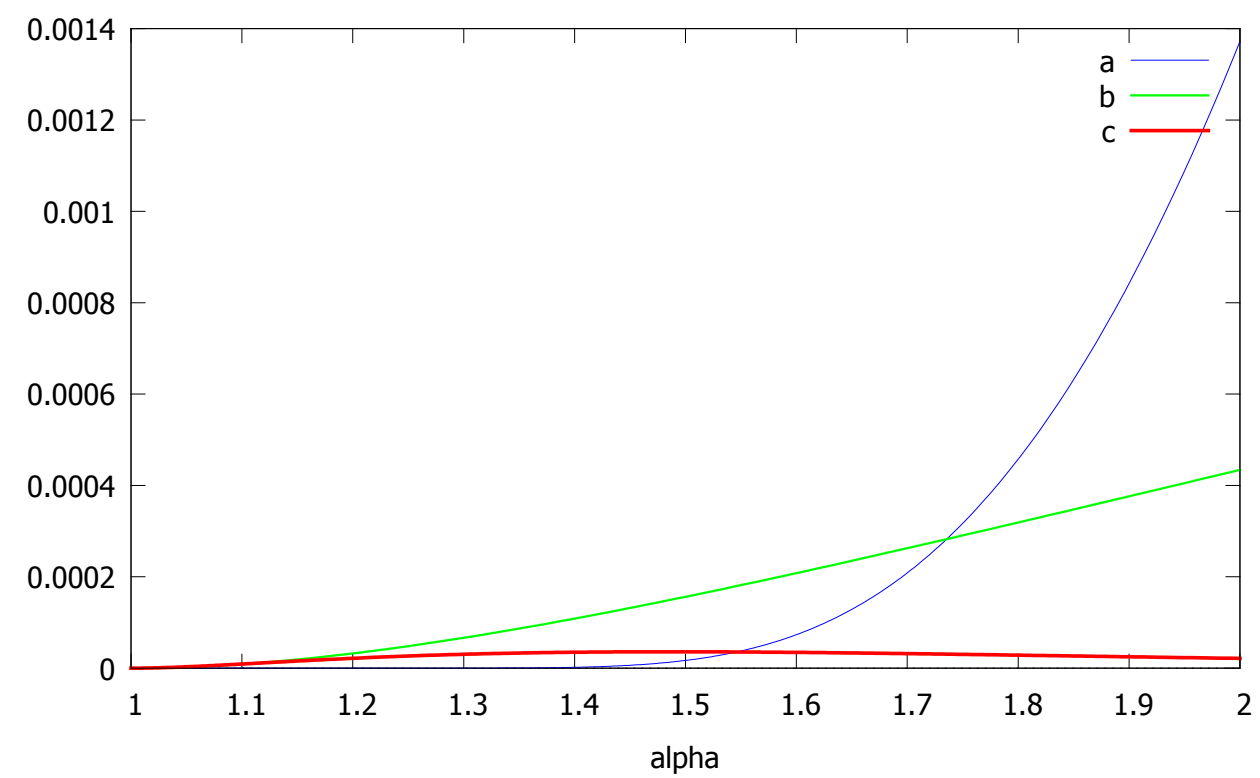

Figure 1. Graphs of constants in Proposition 2 for $\alpha \in(1,2)$ : (a) $\left(\frac{1}{9}\right)^{\frac{\alpha+1}{\alpha-1}}$; (b) $\left(\frac{\alpha-1}{16(\alpha+1)}\right)^{2} ;\left(\right.$ c) $\left(\frac{\alpha-1}{12(\alpha+1)}\right)^{\alpha+1}$.

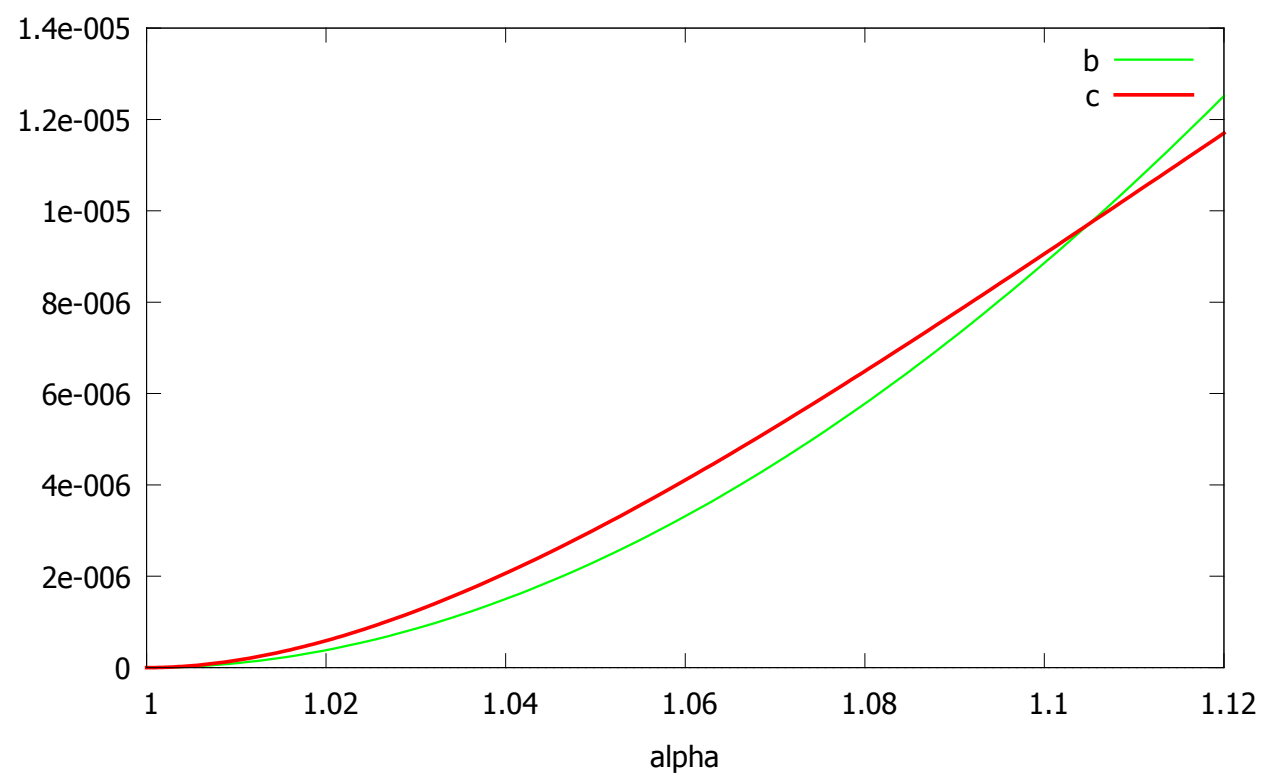

Figure 2. Graphs of constants in Proposition 2 for $\alpha \in(1,1.12)$ : (b) $\left(\frac{\alpha-1}{16(\alpha+1)}\right)^{2}$; (c) $\left(\frac{\alpha-1}{12(\alpha+1)}\right)^{\alpha+1}$.

By symmetry, the right hand side of the above inequality is equal to

$$
2 \int_{0}^{1} \int_{0}^{\frac{1}{2 n}} \frac{\left(f_{n}(x)-f_{n}(y)\right)^{2}}{|x-y|^{1+\alpha}} d x d y
$$


Denote the last double integral by $J_{n}$. We have

$$
J_{n} \leq \int_{0}^{\frac{2}{n}} \int_{0}^{\frac{1}{n}} \frac{\left(f_{n}(x)-f_{n}(y)\right)^{2}}{|x-y|^{1+\alpha}} d x d y+\int_{\frac{2}{n}}^{1} \int_{0}^{\frac{1}{n}} \frac{\left(f_{n}(x)-f_{n}(y)\right)^{2}}{|x-y|^{1+\alpha}} d x d y=I_{n, 1}+I_{n, 2} .
$$

Recall that $f_{n}(x)=f(n x)$, where $f$ is a $C^{\infty}$-class function. Observe that for $x, y \in[0,2]$ we have

$$
\left|f_{n}(x)-f_{n}(y)\right| \leq \sup _{z \in[0,2]}\left|f_{n}^{\prime}(z)\right||x-y|=n \sup _{z \in[0,2]}\left|f^{\prime}(z)\right||x-y| \leq C n|x-y| .
$$

By this we obtain

$$
I_{n, 1}=\int_{0}^{\frac{2}{n}} \int_{0}^{\frac{1}{n}} \frac{\left(f_{n}(x)-f_{n}(y)\right)^{2}}{|x-y|^{1+\alpha}} d x d y \leq C^{2} n^{2} \int_{0}^{\frac{2}{n}} \int_{y-\frac{2}{n}}^{y+\frac{2}{n}}|x-y|^{1-\alpha} d x d y \leq C_{\alpha} n^{\alpha-1} .
$$

Similarly,

$$
I_{n, 2} \leq \int_{\frac{2}{n}}^{1} \int_{0}^{\frac{1}{n}} \frac{1}{|x-y|^{1+\alpha}} d x d y \leq \frac{1}{n} \int_{\frac{2}{n}}^{\infty}\left|\frac{1}{n}-y\right|^{-1-\alpha} d y=C_{\alpha} n^{\alpha-1}
$$

which ends the proof.

Proof of Corollary 1 . Let $b_{0} \in(a, b]$ be such that

$$
f^{2}\left(b_{0}\right) g^{2}\left(b_{0}\right)=\max _{x \in(a, b]} f^{2}(x) g^{2}(x)
$$

We have

$$
\int_{a}^{b} \int_{a}^{b} \frac{(f(x)-f(y))^{2}}{|x-y|^{1+\alpha}} g(x) g(y) d x d y \geq g^{2}\left(b_{0}\right) \int_{a}^{b_{0}} \int_{a}^{b_{0}} \frac{(f(x)-f(y))^{2}}{|x-y|^{1+\alpha}} d x d y,
$$

which, by Theorem 2, is larger than

$$
\frac{C_{\alpha}^{(4)}}{\left(b_{0}-a\right)^{\alpha-1}} f^{2}\left(b_{0}\right) g^{2}\left(b_{0}\right) \geq \frac{C_{\alpha}^{(4)}}{\left(b_{0}-a\right)^{\alpha-1}} \frac{1}{(b-a)} \int_{a}^{b} f^{2}(x) g^{2}(x) d x=\frac{C_{\alpha}^{(4)}}{(b-a)^{\alpha}} \int_{a}^{b} f^{2}(x) g^{2}(x) d x .
$$

\section{Spectral gap estimate}

Proof of Theorem 4. With no loss of generality we provide the arguments for the symmetric interval $(-a, a), 0<a<\infty$, only. Let $V \in \mathcal{V}^{\alpha}((-a, a))$. Recall that the orthonormal basis $\left\{\varphi_{n}\right\}$ is chosen so that $\varphi_{n}$ are either symmetric or antisymmetric. If $\varphi_{2}$ is antisymmetric, then Theorem 4 follows from Theorem 3. Assume now that $\varphi_{2}$ is symmetric. We directly deduce from Theorem 2 that the function $\varphi_{2} / \varphi_{1}$ has a bounded derivative in each interval $\left[a_{0}, b_{0}\right],-a<a_{0}<b_{0}<a$. Hence $\varphi_{2} / \varphi_{1}$ is a Lipschitz function in each interval $\left[a_{0}, b_{0}\right] \subset(-a, a)$. Thus, by Proposition 1 , it is enough to estimate from below the double integral

$$
\int_{-a}^{a} \int_{-a}^{a} \frac{(f(x)-f(y))^{2}}{|x-y|^{1+\alpha}} \varphi_{1}(x) \varphi_{1}(y) d x d y, \quad \text { with } \quad f=\varphi_{2} / \varphi_{1} .
$$

Note that $f$ is symmetric on $(-a, a), f$ changes the sign in $(-a, a)$ and $\int_{-a}^{a} f^{2}(x) \varphi_{1}^{2}(x) d x=1$.

Let $a_{0}=\min \{x \in[0, a): f(x)=0\}$. Consider the following two cases.

(Case 1) Assume that

$$
\int_{a_{0}}^{a} f^{2}(x) \varphi_{1}^{2}(x) d x \geq 1 / 4
$$


We have

$$
\begin{aligned}
\int_{-a}^{a} \int_{-a}^{a} \frac{(f(x)-f(y))^{2}}{|x-y|^{1+\alpha}} \varphi_{1}(x) \varphi_{1}(y) d x d y \geq & \int_{a_{0}}^{a} \int_{a_{0}}^{a} \frac{(f(x)-f(y))^{2}}{|x-y|^{1+\alpha}} \varphi_{1}(x) \varphi_{1}(y) d x d y \\
& +\int_{-a}^{-a_{0}} \int_{-a}^{-a_{0}} \frac{(f(x)-f(y))^{2}}{|x-y|^{1+\alpha}} \varphi_{1}(x) \varphi_{1}(y) d x d y \\
= & 2 \int_{a_{0}}^{a} \int_{a_{0}}^{a} \frac{(f(x)-f(y))^{2}}{|x-y|^{1+\alpha}} \varphi_{1}(x) \varphi_{1}(y) d x d y
\end{aligned}
$$

Let now $b_{0} \in\left[a_{0}, a\right)$ be such that $f^{2}\left(b_{0}\right) \varphi_{1}^{2}\left(b_{0}\right)=\max _{x \in\left(a_{0}, a\right)} f^{2}(x) \varphi_{1}^{2}(x)$. We have

$$
\int_{a_{0}}^{a} \int_{a_{0}}^{a} \frac{(f(x)-f(y))^{2}}{|x-y|^{1+\alpha}} \varphi_{1}(x) \varphi_{1}(y) d x d y \geq \varphi_{1}^{2}\left(b_{0}\right) \int_{a_{0}}^{b_{0}} \int_{a_{0}}^{b_{0}} \frac{(f(x)-f(y))^{2}}{|x-y|^{1+\alpha}} d x d y,
$$

which, by Theorem 2, is larger than

$$
\frac{C_{\alpha}^{(4)}}{\left(b_{0}-a_{0}\right)^{\alpha-1}} f^{2}\left(b_{0}\right) \varphi_{1}^{2}\left(b_{0}\right) \geq \frac{C_{\alpha}^{(4)}}{\left(b_{0}-a_{0}\right)^{\alpha-1}} \frac{1}{\left(a-a_{0}\right)} \int_{a_{0}}^{a} f^{2}(x) \varphi_{1}^{2}(x) d x \geq \frac{1}{4} \frac{C_{\alpha}^{(4)}}{\left(a-a_{0}\right)^{\alpha}} .
$$

It follows that

$$
\int_{-a}^{a} \int_{-a}^{a} \frac{(f(x)-f(y))^{2}}{|x-y|^{1+\alpha}} \varphi_{1}(x) \varphi_{1}(y) d x d y \geq \frac{1}{2} \frac{C_{\alpha}^{(4)}}{(2 a)^{\alpha}}
$$

which ends the proof in the first case.

(Case 2) Suppose now that

$$
\int_{0}^{a_{0}} f^{2}(x) \varphi_{1}^{2}(x) d x \geq 1 / 4
$$

Notice that

$$
\left(\int_{a_{0}}^{a} f(x) \varphi_{1}^{2}(x) d x\right)^{2} \leq \int_{a_{0}}^{a} f^{2}(x) \varphi_{1}^{2}(x) d x \int_{a_{0}}^{a} \varphi_{1}^{2}(x) d x \leq \varphi_{1}^{2}\left(a_{0}\right)\left(a-a_{0}\right) \int_{a_{0}}^{a} f^{2}(x) \varphi_{1}^{2}(x) d x
$$

by Schwarz inequality and Theorem 1, and

$$
-\int_{a_{0}}^{a} f(x) \varphi_{1}^{2}(x) d x=\int_{0}^{a_{0}} f(x) \varphi_{1}^{2}(x) d x
$$

by the fact that $f$ is symmetric and $\int_{-a}^{a} f(x) \varphi_{1}^{2}(x) d x=0$. Observe that without loosing generality we may and do assume that $f \geq 0$ on $\left[0, a_{0}\right]$. Let $a^{*} \in\left[0, a_{0}\right)$ be such that $f\left(a^{*}\right)=\max _{x \in\left[0, a_{0}\right)} f(x)$. Note that $\int_{0}^{a} f^{2}(x) \varphi_{1}^{2}(x) d x=1 / 2$. By (32) and (33), we have

$$
\begin{aligned}
1 / 4 \geq \int_{a_{0}}^{a} f^{2}(x) \varphi_{1}^{2}(x) d x & \geq \frac{\left(\int_{a_{0}}^{a} f(x) \varphi_{1}^{2}(x) d x\right)^{2}}{\varphi_{1}^{2}\left(a_{0}\right)\left(a-a_{0}\right)}=\frac{\left(\int_{0}^{a_{0}} f(x) \varphi_{1}^{2}(x) d x\right)^{2}}{\varphi_{1}^{2}\left(a_{0}\right)\left(a-a_{0}\right)} \\
& =\frac{f^{2}\left(a^{*}\right)\left(\int_{0}^{a_{0}} f(x) \varphi_{1}^{2}(x) d x\right)^{2}}{f^{2}\left(a^{*}\right) \varphi_{1}^{2}\left(a_{0}\right)\left(a-a_{0}\right)} \geq \frac{\left(\int_{0}^{a_{0}} f^{2}(x) \varphi_{1}^{2}(x) d x\right)^{2}}{f^{2}\left(a^{*}\right) \varphi_{1}^{2}\left(a_{0}\right)\left(a-a_{0}\right)},
\end{aligned}
$$

which implies that

$$
f^{2}\left(a^{*}\right) \varphi_{1}^{2}\left(a_{0}\right) \geq 1 /\left(4\left(a-a_{0}\right)\right) .
$$


We have

$$
\begin{aligned}
\int_{-a}^{a} \int_{-a}^{a} \frac{(f(x)-f(y))^{2}}{|x-y|^{1+\alpha}} \varphi_{1}(x) \varphi_{1}(y) d x d y & \geq \int_{0}^{a_{0}} \int_{0}^{a_{0}} \frac{(f(x)-f(y))^{2}}{|x-y|^{1+\alpha}} \varphi_{1}(x) \varphi_{1}(y) d x d y \\
& +\int_{-a_{0}}^{0} \int_{-a_{0}}^{0} \frac{(f(x)-f(y))^{2}}{|x-y|^{1+\alpha}} \varphi_{1}(x) \varphi_{1}(y) d x d y \\
& =2 \int_{0}^{a_{0}} \int_{0}^{a_{0}} \frac{(f(x)-f(y))^{2}}{|x-y|^{1+\alpha}} \varphi_{1}(x) \varphi_{1}(y) d x d y \\
& \geq 2 \varphi_{1}^{2}\left(a_{0}\right) \int_{a^{*}}^{a_{0}} \int_{a^{*}}^{a_{0}} \frac{(f(x)-f(y))^{2}}{|x-y|^{1+\alpha}} d x d y
\end{aligned}
$$

Now, using Theorem 2 and (34), we obtain

$$
\int_{-a}^{a} \int_{-a}^{a} \frac{(f(x)-f(y))^{2}}{|x-y|^{1+\alpha}} \varphi_{1}(x) \varphi_{1}(y) d x d y \geq 2 \varphi_{1}^{2}\left(a_{0}\right) \frac{C_{\alpha}^{(4)}}{\left(a_{0}-a^{*}\right)^{\alpha-1}} f^{2}\left(a^{*}\right) \geq \frac{1}{2} \frac{C_{\alpha}^{(4)}}{(2 a)^{\alpha}},
$$

which completes the proof.

Acknowledgements. I am deeply indebted to Prof. T. Kulczycki, my supervisor, for his help and guidance in investigating the theory and preparing this paper, which is part of my Ph.D. thesis. Special thanks go to Dr. B. Dyda for his reading of the manuscript, illuminating discussion and pointing me out the papers [34, 39] and the connections between the GRR-Lemma, the inequalities (8) and the embedding theory of Sobolev spaces. Also, I would like to thank Dr. M. Kwaśnicki for his careful reading of the manuscript and helpful suggestions and comments.

\section{REFERENCES}

[1] B. Andrews, J. Clutterbuck, Proof of the fundamental gap conjecture, preprint, 2010, arXiv:1006.1686v2.

[2] L. Arnold, P. Imkeller, Stratonovich calculus with spatial parameters and anticipative problems in multiplicative ergodic theory, Stoch. Proc. Appl. 62 (1), 19-54, 1996.

[3] A. Ashbaugh, R. Benguria, Optimal lower bounds for eigenvalue gaps for Schrödinger operators with symmetric single well potentials and related results, in "Maximum Principles and Eigenvalue Problems in Partial Differential Equations", Longman, White Plains, NY, 1988.

[4] A. Ashbaugh, R. Benguria, Optimal lower bounds for the gaps between the first two eigenvalues of one-dimensional Schrödinger operators with symmetric single well potentials, Proc. Amer. Math. Soc. 105, 1989, 419-424.

[5] R. Bañuelos, T. Kulczycki, The Cauchy process and the Steklov problem, J. Funct. Anal. 211 (2), $2004,355-423$.

[6] R. Bañuelos, T. Kulczycki, Eigenvalue gaps for the Cauchy process and a Poincaré inequality, J. Funct. Anal 234, 2006, 199-225.

[7] R. Bañuelos, T. Kulczycki, Spectral gap for the Cauchy process on convex, symmetric domains, Comm. Partial Differential Equations 31, 2006, 18411878.

[8] R. Bañuelos, T. Kulczycki, Trace estimates for stable processes, Probab. Theory Relat. Fields 142, $2008,313-338$.

[9] R. Bañuelos, T. Kulczycki, P. J. Méndez-Hernández, On the shape of the ground state eigenfunction for stable processes, Potential Anal. 24, 2006, 205-221.

[10] R. Bañuelos, P. J. Méndez-Hernández, Sharp inequalities for heat kernels of Schrödinger operators and applications to spectral gaps, J. Funct. Anal. 176 (2), 2000, 368-399.

[11] M. van den Berg, On condensation in the free-boson gas and the spectrum of the Laplacian, J. Statist. Phys. 31, 1983, 623-637.

[12] R. M. Blumenthal, R. K. Getoor, Markov Processes and Potential Theory, Springer, New York, 1968.

[13] R. M. Blumenthal, R. K. Getoor, D. B. Ray, On the distribution of first hits for the symmetric stable processes, Trans. Amer. Math. Soc. 99, 1961, 540-554.

[14] K. Bogdan, T. Byczkowski, Potential theory for the $\alpha$-stable Schrödinger operator on bounded Lipschitz domain, Studia Math. 133 (1), 1999, 53-92.

[15] K. Bogdan, T. Byczkowski, Potential theory of Schrödinger operator based on fractional Laplacian, Prob. Math. Statist. 20, 2000, 293-335.

[16] K. Bogdan et al, Potential Analysis of Stable processes and its Extensions (ed. P. Graczyk, A. Stós), Lecture Notes in Mathematics 1980, Springer, Berlin, 2009. 
[17] K. Bogdan, T. Jakubowski, Estimates of the Green function for the fractional Laplacian perturbed by gradient, arXiv:1009.2472.

[18] K. Bogdan, T. Kulczycki, A. Nowak, Gradient estimates for harmonic and q-harmonic functions of symmetric stable processes, Illinois J. Math. 46 (2) (2002), 541-556.

[19] K. Bogdan, T. Kulczycki, M. Kwaśnicki, Estimates and structure of $\alpha$-harmonic functions, Prob. Theory Rel. Fields 140 (3-4), 2008, 345-381.

[20] R. Carmona, W. C. Masters, B. Simon, Relativistic Schrödinger operators: asymptotic behaviour of the eigenfunctions, J. Funct. Anal. 91, 1990, 117-142.

[21] Z. Q. Chen, P.J. Fitzsimmons, M. Takeda, J. Ying, T.-S. Zhang, Absolute continuity of symmetric Markov processes, Ann. Probab. 32 (2004), 2067-2098.

[22] Z. Q. Chen, R. Song, General gauge and conditional gauge theorems, Ann. Probab. 30 (2002), no. 3, $1313-1339$.

[23] Z. Q. Chen, R. Song, Intrinsic ultracontractivity and conditional gauge for symmetric stable processes, J. Funct. Anal. 150 (1), 1997, 204-239.

[24] Z. Q. Chen, R. Song, Intrinsic ultracontractivity, conditional lifetimes and conditional gauge for symmetric stable processes on rough domains, Illinois J. Math. 44 (1), 2000, 138-160.

[25] Z. Q. Chen, R. Song, Two sided eigenvalue estimates for subordinate Brownian motion in bounded domains, J. Funct. Anal. 226, 2005, 90-113.

[26] Z. Q. Chen, R. Song, Continuity of eigenvalues of subordinate processes in domains, Math. Z. 252 (2006), 71-89.

[27] K. L. Chung, Z. Zhao, From Brownian Motion to Schrödinger's Equation, Springer, New York, 1995.

[28] B. Davis, On the spectral gap for the fixed mambranes, Ark. Mat. 39 (1), 2001, 65-74.

[29] R. D. DeBlassie, Higher order PDEs and symmetric stable processes, Probab. Theory Relat. Fields 129(4), 495536.

[30] R. D. DeBlassie, P. J. Méndez-Hernández, $\alpha$-continuity properties of the symmetric $\alpha$-stable process, Trans. Amer. Math. Soc. 359, (2007), 2343-2359.

[31] B. Dyda, A fractional order Hardy inequality, Illinois J. Math., 48 (2), 2004, 575-588.

[32] B. Dyda, T. Kulczycki, Spectral gap for stable process on convex planar double symmetric domains, Potential Anal. 27, 2007, 101-132.

[33] M. Fukushima, Y. Oshima, M. Takeda, Dirichlet Forms and Symmetric Markov Processes, 1994, de Gruyter, Berlin.

[34] A. M. Garsia, E. Rodemich, H. Rumsey, A real variable lemma and the continuity of paths of some Gaussian processes, Indiana Univ. Math. J. 20, 565-578, 1970/1971.

[35] F. Hiroshima, T. Ichinose, J. Lörinczi, Path integral representation for Schrödinger operators with Bernstein functions of the Laplacian, arXiv 0906.0103, 2009.

[36] R. L. Frank, E. H. Lieb, R. Seiringer, Hardy-Lieb-Thirring inequalities for fractional Schrödinger operators, J. Amer. Math. Soc. 21 (2008), no. 4, 925-950.

[37] K. Kaleta, T. Kulczycki, Intrinsic ultracontractivity for Schrödinger operators based on fractional Laplacians, Potential Anal. 33 (4), 2010, 313-339.

[38] K. Kaleta, J. Lörinczi, Analytic properties of fractional Schrödinger semigroups and Gibbs measures for symmetric stable processes, arXiv:1011.2713, preprint, 2010.

[39] M. Kassmann, A note on integral inequalities and embeddings of Besov spaces, JIPAM. J. Inequal. Pure Appl. Math., 4 (5): Article 107, 3 pp. (electronic), 2003.

[40] T. Kulczycki, Properties of Green function of symmetric stable processes, Probab. Math. Statist. 17, 1997, 339364 .

[41] T. Kulczycki, Intrinsic ultracontractivity for symmetric stable process, Bull. Polish Acad. Sci. Math. 46 (3), 1998 , 325-334

[42] T. Kulczycki, M. Kwaśnicki, J. Małecki, A. Stós, Spectral properties of the Cauchy process, Proc. London Math. Soc. 101 (2) (2010), 589-622.

[43] T. Kulczycki, B. Siudeja, Intrinsic ultracontractivity of the Feynman-Kac semigroup for the relativistic stable process, Trans. Amer. Math. Soc. 358 (11), 2006, 5025-5057.

[44] M. Kwaśnicki, Spectral gap estimate for stable processes on arbitrary bounded open sets, Probab. Math. Statist. 28 (1), 2008, 163-167.

[45] M. Kwaśnicki, Eigenvalues of the fractional Laplace operator in the interval, arXiv:1012.1133 preprint, 2010.

[46] R. Lavine, The eigenvalue gap for one dimensional convex potentials, Proc. Amer. Math. Soc. 121 (1994), 815-821.

[47] E. H. Lieb, R. Seiringer, The Stability of Matter in Quantum Mechanics, Cambridge University Pr, 2009.

[48] J. Ling, A lower bound for the gap between the first two eigenvalues of Schrödinger operators on convex domains in $S^{n}$ and $\mathbf{R}^{n}$, Michigan Math. J 40 (1993), 259-270.

[49] J. Lörinczi, J. Małecki, Spectral properties of the massless relativistic harmonic oscillator, arXiv:1006.3665, preprint, 2010. 
[50] I. M. Singer, B. Wong, S. T. Yau, S. S. T. Yau, An estimate of the gap of the first two eigenvalues in the Schrödinger operator, Ann. Scuola Norm. Sup. Pisa Cl. Sci. (4) 12 (2), 1985, 319-333.

[51] R. Smits, Spectral gaps and rates to equilibrium for diffusions in convex domains, Michigan Math. J. 43 (1996), 141-157.

[52] Q.-H. Yu, J.-Q. Zhong, Lower bounds of the gap between the first and second eigenvalues of the Schrödinger operator, Trans. Amer. Math. Soc. 294, 1986, 341-349.

[53] Z. Zhao, A probabilistic principle and generalized Schrödinger perturbations, J. Funct. Anal. 101 (1), 1991, 162176.

Kamil Kaleta, Institute of Mathematics and Computer Science, Wroceaw University of Technology, Wyb. Wyspiańskiego 27, 50-370 Wroceaw, Poland

E-mail address: kamil.kaleta@pwr.wroc.pl 